\title{
Analysis and Identification of Multidimensional Singularities using the Continuous Shearlet Transform
}

\author{
Kanghui Guo and Demetrio Labate
}

\begin{abstract}
In this chapter, we illustrate the properties of the continuous shearlet transform with respect to its ability to describe the set of singularities of multidimensional functions and distributions. This is of particular interest since singularities and other irregular structures typically carry the most essential information in multidimensional phenomena. Consider, for example, the edges of natural images or the moving fronts in the solutions of transport equations. In the following, we show that the continuous shearlet transform provides a precise geometrical characterization of the singularity sets of multidimensional functions and precisely characterizes the boundaries of $2 \mathrm{D}$ and $3 \mathrm{D}$ regions through its asymptotic decay at fine scales. These properties go far beyond the continuous wavelet transform and other classical methods, and set the groundwork for very competitive algorithms for edge detection and feature extraction of $2 \mathrm{D}$ and $3 \mathrm{D}$ data.
\end{abstract}

Key words: analysis of singularities, continuous wavelet transform, shearlets, sparsity, wavefront set, wavelets

\section{Introduction}

A well known property of the continuous wavelet transform is its special ability to identify the singularities of functions and distributions. This property is a manifestation of the locality of the wavelet transform, which is extremely sensitive to local regularity structures. Specifically, if $f$ is a function which is smooth apart from a

\section{Kanghui Guo}

Missouri State University, Department of Mathematics, Springfield, MO 65804, USA,

e-mail: KanghuiGuo@MissouriState.edu

Demetrio Labate

University of Houston, Department of Mathematics, Houston, TX 77204, USA,

e-mail: dlabate@math.uh.edu 
discontinuity at a point $x_{0}$, then the continuous wavelet transform of $f$, denoted by $\mathscr{W}_{\psi} f(a, t)$, exhibits very rapid asymptotic decay as $a$ approaches 0 , unless $t$ is near $x_{0}[14,16]$. The complement of the set of locations where $\mathscr{W}_{\psi} f(a, t)$ has rapid asymptotic decay, as $a \rightarrow 0$, is called the singular support of $f$, and it corresponds, essentially, to the set where $f$ is not regular. Indeed, an even finer analysis of the local regularity properties of $f$, expressed in terms of local Lipschitz regularity, can be performed using the wavelet transform. This shows that there is a precise correspondence between the Lipschitz exponent $\alpha$ of $f$ at a point $x_{0}$ (where $\alpha$ measures the regularity type) and the asymptotic behaviour of $\mathscr{W}_{\psi} f\left(a, x_{0}\right)$ as $a \rightarrow 0$ (see $[11,10]$ ). This is in contrast with the traditional Fourier analysis which is only sensitive to global regularity properties and cannot be used to measure the regularity of a function $f$ at a specific location.

However, despite all its good properties, the wavelet transform is unable to provide additional information about the geometry of the set of singularities of $f$. In many situations, such as in the study of the propagation of singularities associated with PDEs or in image processing applications such as edge detection and image restoration and enhancement, it is extremely important not only to identify the location of singularities, but also to capture their geometrical information, such as the orientation and the curvature of discontinuity curves. We will show that, to this purpose, directional multiscale methods such as the shearlet transform provide, in a certain sense, the most effective solution, thanks to their ability to combine the microlocal properties of the wavelet transform and a sharp sensitivity for directional information.

In fact, it was shown that the continuous curvelet and shearlet transforms can be used to characterize the wavefront set of functions and distributions [1, 4, 17]. Furthermore, as will be discussed in this chapter, the continuous shearlet transform can be used to provide a very precise geometric description of the set of singularities, together with the analysis of the singularity type. Not only these results demonstrate that the continuous shearlet transform is a highly effective microanalysis tool, going far beyond the traditional wavelet framework; they also set the theoretical groundwork for very competitive numerical algorithms for edge analysis and detection, which will be discussed in Chapter 8 of this volume (see also the work in [19]).

In order to illustrate the properties of the shearlet transform with respect to the analysis of singularities, let us start by presenting a few simple examples. This will introduce some general concepts which will be further elaborated in the rest of the chapter.

\subsection{Example: Line singularity}

In many signal and image processing applications, there is a particular interest in functions which are well localized, that is, they have rapid decay both in $\mathbb{R}^{n}$ and in the Fourier domain. Since functions which have rapid decay must have highly regular Fourier transform and vice versa, it follows that well localized functions 
must also exhibit good decay both in $\mathbb{R}^{n}$ and in the Fourier domain. Hence, one way to obtain well localized functions is to define them in the Fourier domain so that they have both compact support and high regularity. In particular, we are interested in affine family of functions on $\mathbb{R}^{n}$ of the form $\psi_{M, t}(x)=|\operatorname{det} M|^{-1 / 2} \psi\left(M^{-1}(x-t)\right)$, where $t \in \mathbb{R}^{n}$ and $M \in G L_{n}(\mathbb{R})$. Indeed we have the following observation stating, essentially, that if $\hat{\psi}$ has compact support and high regularity, then the functions $\psi_{M, t}$ are well localized.

Proposition 1. Suppose that $\psi \in L^{2}\left(\mathbb{R}^{n}\right)$ is such that $\hat{\psi} \in C_{c}^{\infty}(R)$, where $R=$ supp $\hat{\psi} \subset \mathbb{R}^{n}$. Then, for each $k \in \mathbb{N}$, there is a constant $C_{k}>0$ such that, for any $x \in \mathbb{R}^{n}$, we have

$$
\left|\psi_{M, t}(x)\right| \leq C_{k}|\operatorname{det} M|^{-\frac{1}{2}}\left(1+\left|M^{-1}(x-t)\right|^{2}\right)^{-k} .
$$

In particular, $C_{k}=k m(R)\left(\|\hat{\psi}\|_{\infty}+\left\|\triangle^{k} \hat{\psi}\right\|_{\infty}\right)$, where $\triangle=\sum_{i=1}^{n} \frac{\partial^{2}}{\partial \xi_{i}^{2}}$ is the frequency domain Laplacian operator and $m(R)$ is the Lebesgue measure of $R$.

Proof. From the definition of the Fourier transform, it follows that, for every $x \in \mathbb{R}^{n}$,

$$
|\psi(x)| \leq m(R)\|\hat{\psi}\|_{\infty} .
$$

An integration by parts shows that

$$
\int_{R} \triangle \hat{\psi}(\xi) e^{2 \pi i\langle\xi, x\rangle} d \xi=-(2 \pi)^{2}|x|^{2} \psi(x)
$$

and thus, for every $x \in \mathbb{R}^{n}$,

$$
(2 \pi|x|)^{2 k}|\psi(x)| \leq m(R)\left\|\triangle^{k} \hat{\psi}\right\|_{\infty} .
$$

Using (1) and (2), we have

$$
\left(1+(2 \pi|x|)^{2 k}\right)|\psi(x)| \leq m(R)\left(\|\hat{\psi}\|_{\infty}+\left\|\triangle^{k} \hat{\psi}\right\|_{\infty}\right) .
$$

Observe that, for each $k \in \mathbb{N}$,

$$
\left(1+|x|^{2}\right)^{k} \leq\left(1+(2 \pi)^{2}|x|^{2}\right)^{k} \leq k\left(1+(2 \pi|x|)^{2 k}\right) .
$$

Using this last inequality and (3), we have that for each $x \in \mathbb{R}^{n}$

$$
|\psi(x)| \leq k m(R)\left(1+|x|^{2}\right)^{-k}\left(\|\hat{\psi}\|_{\infty}+\left\|\triangle^{k} \hat{\psi}\right\|_{\infty}\right) .
$$

The proof is completed using a simple change of variables.

It is clear that Proposition 1 applies, in particular, to the continuous shearlet systems $\left\{\psi_{a, s, t}=\psi_{M_{a s}, t}\right\}$, where $M_{a s}=\left(\begin{array}{cc}a & -a^{1 / 2} s \\ 0 & a^{1 / 2}\end{array}\right)$, for $a>0, s \in \mathbb{R}, t \in \mathbb{R}^{2}$ (provided that $\psi$ satisfies the assumptions of the Proposition). 

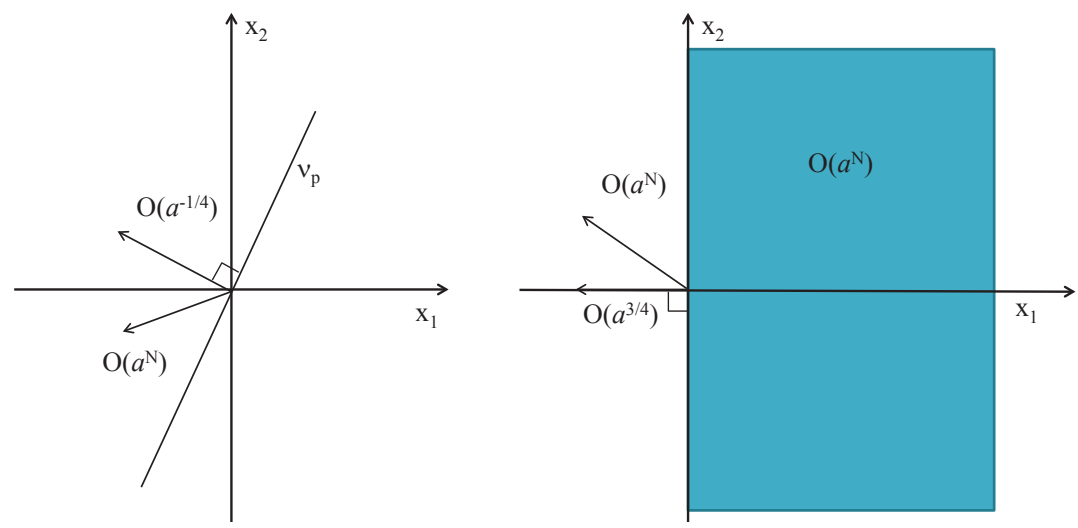

Fig. 1 Linear singularity. Left: The continuous shearlet transform of the linear delta distribution $v_{p}$ has rapid asymptotic decay except when the location variable $t$ is on the support of $v_{p}$ and the shearing variable $s$ corresponds to the normal direction at $t$; in this case $\mathscr{S}_{\mathscr{H}} v_{p}(a, s, t) \sim a^{-1 / 4}$, as $a \rightarrow 0$. Right: The continuous shearlet transform of the Heaviside function has rapid asymptotic decay except when the location variable $t$ is on the line of $x_{1}=0$ and the shearing variable $s$ corresponds to the normal direction to this line; in this case $\mathscr{S}_{\mathscr{H}} v_{p}(a, s, t) \sim a^{3 / 4}$, as $a \rightarrow 0$.

In the following, we will assume that $\psi$, the generator of the continuous shearlet system, is a classical shearlet, according to the definition in Chapter 1 of this volume. That is, for $\xi=\left(\xi_{1}, \xi_{2}\right) \in \mathbb{R}^{2}, \xi_{2} \neq 0$, we have

$$
\hat{\psi}(\xi)=\hat{\psi}_{1}\left(\xi_{1}\right) \hat{\psi}_{2}\left(\frac{\xi_{2}}{\xi_{1}}\right)
$$

where

- $\hat{\psi}_{1} \in C_{c}^{\infty}(\mathbb{R})$, supp $\hat{\psi}_{1} \subset\left[-2,-\frac{1}{2}\right] \cup\left[\frac{1}{2}, 2\right]$, and it satisfies the Calderòn condition $\int_{0}^{\infty}\left|\hat{\psi}_{1}(a \omega)\right|^{2} \frac{d a}{a}=1$, for a.e. $\omega \in \mathbb{R}$;

- $\hat{\psi}_{2} \in C_{c}^{\infty}(\mathbb{R}), \operatorname{supp} \hat{\psi}_{2} \subset\left[-\frac{\sqrt{2}}{4}, \frac{\sqrt{2}}{4}\right]$, and $\left\|\psi_{2}\right\|_{2}=1$.

Since $\hat{\psi} \in C_{c}^{\infty}\left(\mathbb{R}^{2}\right)$, it follows that $\psi \in \mathscr{S}\left(\mathbb{R}^{2}\right)$ and, therefore, the continuous shearlet transform of $f$, denoted by 


$$
\mathscr{S} \mathscr{H}_{\psi} f(a, s, t)=\left\langle f, \psi_{a, s, t}\right\rangle, \quad(a, s, t) \in \mathbb{R}_{+} \times \mathbb{R}, \times \mathbb{R}^{2},
$$

is well defined for all tempered distributions $f \in \mathscr{S}^{\prime}$. Hence, we can examine the continuous shearlet transform of the delta distribution supported along the line $x_{1}=$ $-p x_{2}$, which we denote by $v_{p}\left(x_{1}, x_{2}\right)=\delta\left(x_{1}+p x_{2}\right), p \in \mathbb{R}$ and is defined by

$$
\left\langle v_{p}, f\right\rangle=\int_{\mathbb{R}} f\left(-p x_{2}, x_{2}\right) d x_{2} .
$$

The following simple result from [17] shows that $\mathscr{S}_{\mathcal{H}} v_{p}(a, s, t)$, the continuous shearlet transform of $v_{p}$, has rapid asymptotic decay, as $a \rightarrow 0$, for all values of $s$ and $t$, except when $t$ is on the singularity line and $s$ corresponds to the normal orientation to the singularity line (see Fig. 1). Here and in the following, by rapid asymptotic decay, we mean that, given any $N \in \mathbb{N}$, there is a $C_{N}>0$ such that $\left|\mathscr{S} \mathscr{H}_{\psi} v_{p}(a, s, p)\right| \leq C_{N} a^{N}$, as $a \rightarrow 0$.

Proposition 2. If $t_{1}=-p t_{2}$ and $s=p$, we have

$$
\lim _{a \rightarrow 0} a^{\frac{1}{4}} \mathscr{S} \mathscr{H}_{\psi} v_{p}(a, s, t) \neq 0 .
$$

In all other cases,

$$
\lim _{a \rightarrow 0} a^{-N} \mathscr{S} \mathscr{H}_{\psi} v_{p}(a, s, t)=0, \quad \text { for all } N>0 .
$$

Proof. Recall that the Fourier transform of $v_{p}$ is given by

$$
\begin{aligned}
\hat{v}_{p}\left(\xi_{1}, \xi_{2}\right) & =\iint \delta\left(x_{1}+p x_{2}\right) e^{-2 \pi i\langle\xi, x\rangle} d x_{2} d x_{1} \\
& =\int e^{-2 \pi i x_{2}\left(\xi_{2}-p \xi_{1}\right)} d x_{2}=\delta\left(\xi_{2}-p \xi_{1}\right)=v_{\left(-\frac{1}{p}\right)}\left(\xi_{1}, \xi_{2}\right) .
\end{aligned}
$$

That is, the Fourier transform of the linear delta on $\mathbb{R}^{2}$ is another linear delta on $\mathbb{R}^{2}$, where the slope $-\frac{1}{p}$ is replaced by the slope $p$. Hence, a direct computation gives:

$$
\begin{aligned}
\mathscr{S}_{\mathscr{H}_{\psi}} v_{p}(a, s, t) & =\left\langle\hat{v}_{p}, \hat{\psi}_{a, s, t}\right\rangle \\
& =\int_{\mathbb{R}} \hat{\psi}_{a, s, t}\left(\xi_{1}, p \xi_{1}\right) d \xi_{1} \\
& =a^{\frac{3}{4}} \int_{\mathbb{R}} \hat{\psi}\left(a \xi_{1}, \sqrt{a} p \xi_{1}-\sqrt{a} s \xi_{1}\right) e^{-2 \pi i \xi_{1}\left(t_{1}+p t_{2}\right)} d \xi_{1} \\
& =a^{-\frac{1}{4}} \int_{\mathbb{R}} \hat{\psi}\left(\xi_{1}, a^{-\frac{1}{2}} p \xi_{1}-a^{-\frac{1}{2}} s \xi_{1}\right) e^{-2 \pi i a^{-1} \xi_{1}\left(t_{1}+p t_{2}\right)} d \xi_{1} \\
& =a^{-\frac{1}{4}} \int_{\mathbb{R}} \hat{\psi}_{1}\left(\xi_{1}\right) \hat{\psi}_{2}\left(a^{-\frac{1}{2}}(p-s)\right) e^{-2 \pi i a^{-1} \xi_{1}\left(t_{1}+p t_{2}\right)} d \xi_{1} \\
& =a^{-\frac{1}{4}} \hat{\psi}_{2}\left(a^{-\frac{1}{2}}(p-s)\right) \psi_{1}\left(-a^{-1}\left(t_{1}+p t_{2}\right)\right) .
\end{aligned}
$$


Recall that $\hat{\psi}_{2}$ is compactly supported in the interval $[-1,1]$. It follows that, if $s \neq p$, then $\hat{\psi}_{2}\left(a^{-1 / 2}(p-s)\right) \rightarrow 0$ as $a \rightarrow 0$ since $|p-s|>\sqrt{a}$ for $a$ sufficiently small. Thus $\lim _{a \rightarrow 0} \mathscr{S}^{H} v_{\psi} v_{p}(a, s, t)=\lim _{a \rightarrow 0}\left\langle\hat{v}_{p}, \hat{\psi}_{a, s, t}\right\rangle=0$ for $s \neq p$. On the other hand, if $t_{1}=-p t_{2}$ and $s=p$, then $\hat{\psi}_{2}\left(a^{-1 / 2}(p-s)\right)=\hat{\psi}_{2}(0) \neq 0$, and

$$
\left\langle\hat{v}_{p}, \hat{\psi}_{a, s, t}\right\rangle=a^{-\frac{1}{4}} \hat{\psi}_{2}\left(a^{-\frac{1}{2}}(p-s)\right) \psi_{1}(0) \sim a^{-\frac{1}{4}}, \text { as } a \rightarrow 0 .
$$

Finally, if $t_{1} \neq-p t_{2}$, Proposition 1 implies that, for all $N \in \mathbb{N}$,

$$
\begin{aligned}
& \left\langle\hat{v}_{p}, \hat{\psi}_{a, s, t}\right\rangle \\
& \leq a^{-\frac{1}{4}} \hat{\psi}_{2}\left(a^{-\frac{1}{2}}(p-s)\right)\left|\psi_{1}\left(a^{-1}\left(t_{1}+p t_{2}\right)\right)\right| \\
& \leq C_{N} a^{-\frac{1}{4}} \hat{\psi}_{2}\left(a^{-\frac{1}{2}}(p-s)\right)\left(1+a^{-2}\left(t_{1}+p t_{2}\right)^{2}\right)^{-N} \sim a^{2 N-\frac{1}{4}}, \text { as } a \rightarrow 0 . \square
\end{aligned}
$$

As another example of line singularity, we will consider the Heaviside function $H\left(x_{1}, x_{2}\right)=\chi_{x_{1}>0}\left(x_{1}, x_{2}\right)$. Also in this case, as the following result shows, the continuous shearlet transform has rapid asymptotic decay, as $a \rightarrow 0$, for all values of $s$ and $t$, except when $t$ is on the singularity line and $s$ corresponds to the normal orientation to the singularity line (see Fig. 1). Notice that, using an appropriate change of variables, this result can be extended to deal with step discontinuities along lines with arbitrary orientations.

Proposition 3. If $t=\left(0, t_{2}\right)$ and $s=0$, we have

$$
\lim _{a \rightarrow 0} a^{-\frac{3}{4}} \mathscr{S}_{\mathcal{H}} H\left(a, 0,\left(0, t_{2}\right)\right) \neq 0 .
$$

In all other cases,

$$
\lim _{a \rightarrow 0} a^{-N} \mathscr{S} \mathscr{H}_{\psi} H(a, s, t)=0, \quad \text { for all } N>0 .
$$

Proof. Observe that $\frac{\partial}{\partial x_{1}} H=\delta_{1}$, where $\delta_{1}$ is the delta distribution defined by

$$
\left\langle\delta_{1}, \phi\right\rangle=\int \phi\left(0, x_{2}\right) d x_{2}
$$

and $\phi$ is a function in the Schwartz class $\mathscr{S}\left(\mathbb{R}^{2}\right)$ (notice that here we use the notation of the inner product $\langle$,$\rangle to denote the functional on \mathscr{S}$ ). Hence

$$
\widehat{H}\left(\xi_{1}, \xi_{2}\right)=\left(2 \pi i \xi_{1}\right)^{-1} \hat{\delta}_{1}\left(\xi_{1}, \xi_{2}\right)
$$

where $\hat{\delta}_{1}$ is the distribution obeying

$$
\left\langle\hat{\delta}_{1}, \hat{\phi}\right\rangle=\int \hat{\phi}\left(\xi_{1}, 0\right) d \xi_{1}
$$

The continuous shearlet transform of $H$ can now be expressed as 


$$
\begin{aligned}
\mathscr{S} \mathscr{H}_{\psi} H(a, s, t) & =\left\langle H, \psi_{a, s, t}\right\rangle \\
& =\int_{\mathbb{R}^{2}}\left(2 \pi i \xi_{1}\right)^{-1} \hat{\delta}_{1}(\xi) \overline{\hat{\psi}_{a, s, t}}(\xi) d \xi \\
& =\int_{\mathbb{R}}\left(2 \pi i \xi_{1}\right)^{-1} \overline{\hat{\psi}_{a, s, t}}\left(\xi_{1}, 0\right) d \xi_{1} \\
& =\int_{\mathbb{R}} \frac{a^{3 / 4}}{2 \pi i \xi_{1}} \overline{\hat{\psi}_{1}}\left(a \xi_{1}\right) \overline{\hat{\psi}_{2}}\left(a^{-\frac{1}{2}} s\right) e^{2 \pi i \xi_{1} t_{1}} d \xi_{1} \\
& =\frac{a^{3 / 4}}{2 \pi i} \overline{\hat{\psi}_{2}}\left(a^{-\frac{1}{2}} s\right) \int_{\mathbb{R}} \overline{\hat{\psi}}_{1}(u) e^{2 \pi i u} \frac{t_{1}}{a} \frac{d u}{u},
\end{aligned}
$$

where $t_{1}$ is the first component of $t \in \mathbb{R}^{2}$.

Notice that, by the properties of $\psi_{1}$, the function $\tilde{\psi}_{1}(v)=\int_{\mathbb{R}} \hat{\psi}_{1}(u) e^{2 \pi i u v} \frac{d u}{u}$ decays rapidly, asymptotically, as $v \rightarrow \infty$. Hence, if $t_{1} \neq 0$, it follows that $\tilde{\psi}_{1}\left(\frac{t_{1}}{a}\right)$ decays rapidly, asymptotically, as $a \rightarrow 0$, and, as a result, $\mathscr{S} \mathscr{H}{ }_{\psi} H(a, s, t)$ also has rapid decay as $a \rightarrow 0$. Similarly, by the support conditions of $\hat{\psi}_{2}$, if $s \neq 0$ it follows that the function $\hat{\psi}_{2}\left(a^{-\frac{1}{2}} s\right)$ approaches 0 as $a \rightarrow 0$. Finally, if $t_{1}=s=0$, then

$$
a^{-3 / 4} \mathscr{S}_{\mathscr{H}} \psi\left(a, 0,\left(0, t_{2}\right)\right)=\frac{1}{2 \pi i} \overline{\hat{\psi}}_{2}(0) \int_{\mathbb{R}} \overline{\hat{\psi}}_{1}(u) \frac{d u}{u} \neq 0 .
$$

\subsection{General singularities}

The two examples presented above show that the continuous shearlet transform describes, through its asymptotic decay at fine scales, both the location and the orientation of delta-type and step-type singularities along lines. As will be discussed below, this result holds in much greater generality. In particular, away from the singularities, the continuous shearlet transform has always rapid asymptotic decay. At the singularity, as the examples suggest, the behaviour depends (i) on singularity type and (ii) on the geometry of the singularity set.

For the dependence on the singularity type, both examples show that non-rapid decay occurs at the singularity, in the normal orientation. However, for the step singularity, we found that, as $a \rightarrow 0, \mathscr{S}_{\mathscr{H}} H\left(a, 0,\left(0, t_{2}\right)\right) \sim a^{3 / 4}$ (slow decay), whereas for the delta singularity we found $\mathscr{S}_{\mathcal{H}} v_{p}(a, s, t) \sim a^{-1 / 4}$ (increase). This shows that the sensitivity on the singularity type is consistent with the wavelet analysis as presented in $[10,11]$. Recall, in fact, that if $f \in L^{2}(\mathbb{R})$ is uniformly Lipschitz $\alpha$ in a neighborhood of $t$ and $\tilde{\psi}$ is a nice wavelet, the continuous wavelet transform of $f$ satisfies

$$
\mathscr{W}_{\tilde{\psi}} f(a, t) \leq C a^{\alpha+1 / 2}
$$

which shows that the decay is controlled by the regularity of $f$ at $t$. This analysis extends to the case where $f$ has a jump or delta singularity at $t$, corresponding to $\alpha=0$ and $\alpha=-1$, respectively. This yields that $\mathscr{W}_{\tilde{\psi}} f(a, t)$ has slow decay $a^{1 / 2}$, if 
$t$ is a jump discontinuity, while it increases as $a^{-1 / 2}$ if $t$ is a delta-type singularity (cf. [15]).

That the qualitative behaviours of the decay of the continuous shearlet transform at the singularity is similar to the continuous wavelet transform should not be surprising since the continuous shearlet transform preserves the microlocal features of the continuous wavelet transform. Additional observations about this aspect are found in $[5,17]$.

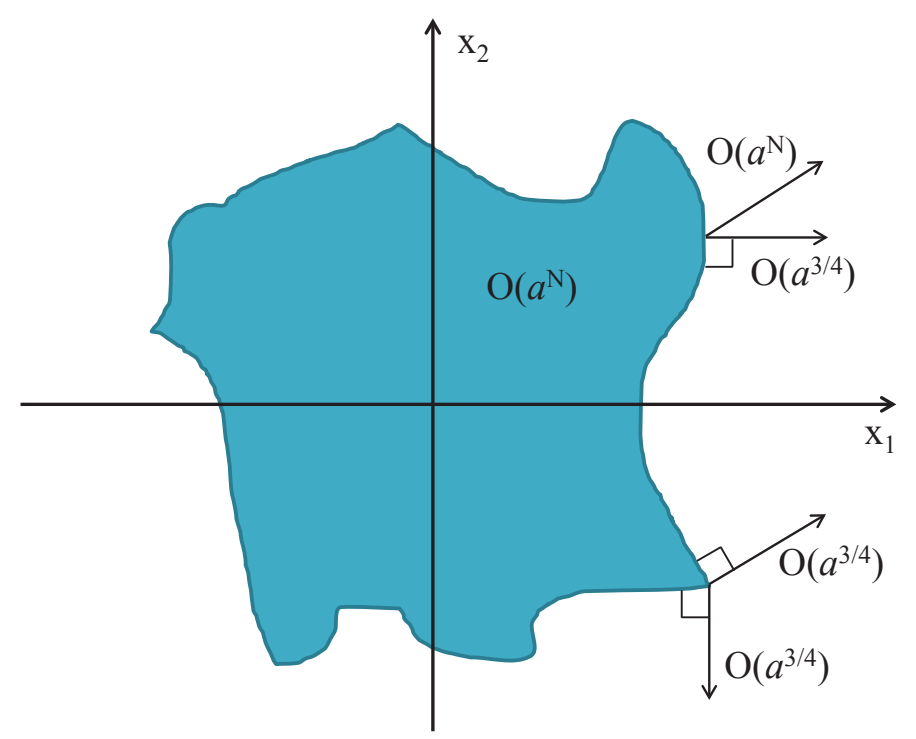

Fig. 2 General region $S$ with piecewise smooth boundary $\partial S$. The continuous shearlet transform of $B=\chi_{S}$ has rapid asymptotic decay except when the location variable $t$ is on $\partial S$ and the shearing variable $s$ corresponds to the normal direction at $t$; in this case $\mathscr{S}_{\mathcal{H}} B(a, s, t) \sim a^{3 / 4}$, as $a \rightarrow 0$. The same slow decay rate occurs at the corner points, for normal orientations.

On the other hand, the ability of the continuous shearlet transform to detect the geometry of the singularity set goes far beyond the continuous wavelet transform and is its most distinctive feature. As a particular manifestation of this ability, we will shows that the continuous shearlet transform provides a very general and elegant characterization of step discontinuities along 2D piecewise smooth curves, which can summarized as follows (see [7,6]). Let $B=\chi_{S}$, where $S \subset \mathbb{R}^{2}$ and its boundary $\partial S$ is a piecewise smooth curve.

- If $t \notin \partial S$, then $\mathscr{S} \mathscr{H}_{\psi} B(a, s, t)$ has rapid asymptotic decay, as $a \rightarrow 0$, for each $s \in \mathbb{R}$. 
- If $t \in \partial S$ and $\partial S$ is smooth near $t$, then $\mathscr{S} \mathscr{H} \psi(a, s, t)$ has rapid asymptotic decay, as $a \rightarrow 0$, for each $s \in \mathbb{R}$ unless $s=s_{0}$ is the normal orientation to $\partial S$ at $p$. In this last case, $\mathscr{S} \mathscr{H}_{\psi} B\left(a, s_{0}, t\right) \sim a^{\frac{3}{4}}$, as $a \rightarrow 0$.

- If $t$ is a corner point of $\partial S$ and $s=s_{0}, s=s_{1}$ are the normal orientations to $\partial S$ at $t$, then $\mathscr{S} \mathscr{H}_{\psi} B\left(a, s_{0}, t\right), \mathscr{S}_{\mathscr{H}} B\left(a, s_{1}, t\right) \sim a^{\frac{3}{4}}$, as $a \rightarrow 0$. For all other orientations, the asymptotic decay of $\mathscr{S} \mathscr{H}_{\psi} B(a, s, t)$ is faster (even if not necessarily "rapid").

This behaviour is illustrated in Fig. 2 .

The proof of this result and its generalization to higher dimensions will be the main content of the sections below.

\section{Analysis of Step Singularities (2D)}

In this section, we describe how the shearlet framework is employed to provide a characterization of the step singularities associated with functions of the form $B=\chi_{S}$, where $S$ is a bounded region in $\mathbb{R}^{2}$, and the boundary set $\partial S$ is piecewise smooth.

Before presenting this result, let us discuss the properties of the continuous shearlet transform that are needed for this analysis. As already noticed in Chapter 1 of this volume, the continuous shearlet transform exhibits a directional bias which is manifested, in particular, by the fact that the shearlet detection of the line singularity given by Proposition 2 does not cover the case where the singularity line is $t_{2}=0$. In this case, in fact, to be able to capture the correct orientation, the shearing variable should be taken in the limit value $s \rightarrow \infty$. As also discussed in Chapter 1 of this volume, this limitation can be overcome by using the cone-adapted continuous shearlet systems.

Hence, for $\xi=\left(\xi_{1}, \xi_{2}\right) \in \mathbb{R}^{2}$, we let $\psi^{(h)}, \psi^{(v)}$ be defined by

$$
\hat{\psi}^{(h)}\left(\xi_{1}, \xi_{2}\right)=\hat{\psi}_{1}\left(\xi_{1}\right) \hat{\psi}_{2}\left(\frac{\xi_{2}}{\xi_{1}}\right), \quad \hat{\psi}^{(h)}\left(\xi_{1}, \xi_{2}\right)=\hat{\psi}_{1}\left(\xi_{2}\right) \hat{\psi}_{2}\left(\frac{\xi_{1}}{\xi_{2}}\right),
$$

and, for

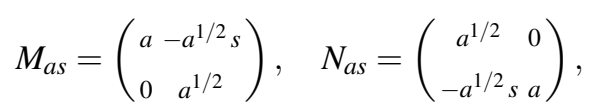

we define the systems of "horizontal" and "vertical" continuous shearlets by

$$
\begin{aligned}
& \left\{\psi_{a, s, t}^{(h)}=\left|\operatorname{det} M_{a s}\right|^{-\frac{1}{2}} \psi^{(h)}\left(M_{a s}^{-1}(x-t)\right): 0<a \leq \frac{1}{4},-\frac{3}{2} \leq s \leq \frac{3}{2}, t \in \mathbb{R}^{2}\right\}, \\
& \left\{\psi_{a, s, t}^{(v)}=\left|\operatorname{det} N_{a s}\right|^{-\frac{1}{2}} \psi^{(h)}\left(N_{a s}^{-1}(x-t)\right): 0<a \leq \frac{1}{4},-\frac{3}{2} \leq s \leq \frac{3}{2}, t \in \mathbb{R}^{2}\right\} .
\end{aligned}
$$

Notice that, in the new definition, the shearing variable is now allowed to vary over a compact interval only. In fact, as also described in Chapter 1 of this volume, each system of continuous shearlets spans only a subspace of $L^{2}\left(\mathbb{R}^{2}\right)$, namely, the spaces $L^{2}\left(\mathscr{P}^{(h)}\right)^{\vee}$ and $L^{2}\left(\mathscr{P}^{(v)}\right)^{\vee}$, where $\mathscr{P}^{(h)}$ and $\mathscr{P}^{(v)}$ are the horizontal and vertical 
cones in the frequency domain, given by

$$
\begin{aligned}
& \mathscr{P}^{(h)}=\left\{\left(\xi_{1}, \xi_{2}\right) \in \mathbb{R}^{2}:\left|\xi_{1}\right| \geq 2 \text { and }\left|\frac{\xi_{2}}{\xi_{1}}\right| \leq 1\right\} \\
& \mathscr{P}^{(v)}=\left\{\left(\xi_{1}, \xi_{2}\right) \in \mathbb{R}^{2}:\left|\xi_{1}\right| \geq 2 \text { and }\left|\frac{\xi_{2}}{\xi_{1}}\right|>1\right\} .
\end{aligned}
$$

Hence, we will define a continuous shearlet transform which uses as analyzing elements either the "horizontal" or the "vertical" continuous shearlet system. That is, for $0<a \leq 1 / 4, s \in \mathbb{R}, t \in \mathbb{R}^{2}$, we define the (fine-scale) cone-adapted continuous shearlet transform as the mapping from $f \in L^{2}\left(\mathbb{R}^{2} \backslash[-2,2]^{2}\right)^{\vee}$ into $\mathscr{S} \mathscr{H}_{\psi} f$ which is given by:

$$
\mathscr{S} \mathscr{H}_{\psi} f(a, s, t)= \begin{cases}\mathscr{S}_{\mathscr{H}_{\psi}^{(h)}}^{(h)} f(a, s, t)=\left\langle f, \psi_{a, s, t}^{(h)}\right\rangle, & \text { if }|s| \leq 1 \\ \mathscr{S}_{\mathcal{H}_{\psi}^{(v)}}^{(v)} f\left(a, \frac{1}{s}, t\right)=\left\langle f, \psi_{a, s, t}^{(v)}\right\rangle, & \text { if }|s|>1 .\end{cases}
$$

The term fine-scale refers to the fact that this shearlet transform is only defined for values $0<a \leq 1 / 4$. In fact, for the analysis of the boundaries of planar regions, we will only be interested in deriving asymptotic estimates as $a$ approaches 0 .

Finally, we will still assume the shearlet generators $\psi^{(h)}, \psi^{(v)}$ are well localized functions. However a few additional assumptions with respect to Sec. 1.1 are needed. For completeness, we summarize below the complete set of assumptions on the functions $\psi_{1}, \psi_{2}$. We assume that:

- $\hat{\psi}_{1} \in C_{c}^{\infty}(\mathbb{R}), \operatorname{supp} \hat{\psi}_{1} \subset\left[-2,-\frac{1}{2}\right] \cup\left[\frac{1}{2}, 2\right]$, is odd, nonnegative on $\left[\frac{1}{2}, 2\right]$ and it satisfies $\int_{0}^{\infty}\left|\hat{\psi}_{1}(a \xi)\right|^{2} \frac{d a}{a}=1$, for a.e. $\xi \in \mathbb{R}$;

- $\hat{\psi}_{2} \in C_{c}^{\infty}(\mathbb{R}), \operatorname{supp} \hat{\psi}_{2} \subset\left[-\frac{\sqrt{2}}{4}, \frac{\sqrt{2}}{4}\right]$, is even, nonnegative, decreasing in $\left[0, \frac{\sqrt{2}}{4}\right)$, and $\left\|\psi_{2}\right\|_{2}=1$.

Before presenting the proof of the general characterization result, it is useful to examine the situation where $S$ is a disc. In this case, thanks to the simpler geometry, it is possible to use a more direct argument than in the general case.

\subsection{Shearlet analysis of circular edges}

Let $D_{R}$ be the ball in $\mathbb{R}^{2}$ of radius $R \geq R_{0}>0$, centered at the origin, and let $B_{R}=$ $\chi_{D_{R}}$. It was shown in [6] (by refining an incomplete proof in [17]) that the continuous shearlet transform of $B_{R}$ exactly characterizes the curve $\partial D_{R}$.

Theorem 1. Let $t \in P=\left\{t=\left(t_{1}, t_{2}\right) \in \mathbb{R}^{2}:\left|\frac{t_{2}}{t_{1}}\right| \leq 1\right\}$.

If $t=t_{0}=R\left(\cos \theta_{0}, \sin \theta_{0}\right)$, for some $\left|\theta_{0}\right| \leq \frac{\pi}{4}$, then

$$
\lim _{a \rightarrow 0^{+}} a^{-\frac{3}{4}} \mathscr{S}_{\mathscr{H}_{\psi}} B_{R}\left(a, \tan \theta_{0}, t_{0}\right) \neq 0
$$


If $t=t_{0}$ and $s \neq \tan \theta_{0}$, or if $t \notin \partial D(0, R)$, then

$$
\lim _{a \rightarrow 0^{+}} a^{-N} \mathscr{S}_{\mathscr{H}} B_{R}(a, s, t)=0, \quad \text { for all } N>0 .
$$

Proof (Sketch). We only sketch the main ideas of this proof, since this result is a special case of Theorem 2, which will be presented below. Also, we will consider the system of horizontal shearlets $\left\{\psi_{a, s, t}^{(h)}\right\}$ only, since the analysis for the vertical shearlets is essentially the same. For simplicity of notation, we will drop the upperscript (h).

A direct computation gives:

$$
\begin{aligned}
& \mathscr{S} \mathscr{H}_{\psi} B_{R}(a, s, t)=\left\langle\widehat{B}_{R}, \widehat{\psi}_{a, s, t}\right\rangle \\
& \quad=a^{\frac{3}{4}} \int_{\mathbb{R}} \int_{\mathbb{R}} \hat{\psi}_{1}\left(a \xi_{1}\right) \hat{\psi}_{2}\left(a^{-\frac{1}{2}}\left(\frac{\xi_{2}}{\xi_{1}}-s\right)\right) e^{2 \pi i\langle\xi, t\rangle} \widehat{B}_{R}\left(\xi_{1}, \xi_{2}\right) d \xi_{1} d \xi_{2} .
\end{aligned}
$$

For $R=1$, the Fourier transform $\widehat{B}_{1}\left(\xi_{1}, \xi_{2}\right)$ is the radial function:

$$
\widehat{B}_{1}\left(\xi_{1}, \xi_{2}\right)=|\xi|^{-1} J_{1}(2 \pi|\xi|)
$$

where $J_{1}$ is the Bessel function of order 1 , whose asymptotic behaviour satisfies [18]:

$$
J_{1}(2 \pi|\xi|)=\frac{1}{\pi}|\xi|^{-\frac{1}{2}} \cos \left(2 \pi|\xi|-\frac{3 \pi}{4}\right)+O\left(|\xi|^{-3 / 2}\right) \quad \text { as }|\xi| \rightarrow \infty .
$$

It is useful to express the integral (10) using polar coordinates. For $\left|\frac{t_{2}}{t_{1}}\right| \leq 1,|s| \leq \frac{3}{2}$ and $\frac{1}{2} R \leq r \leq 2 R$, we write $t=\left(t_{1}, t_{2}\right)$ as $r\left(\cos \theta_{0}, \sin \theta_{0}\right)$, where $0 \leq\left|\theta_{0}\right| \leq \frac{\pi}{4}$. Thus, we have:

$$
\begin{aligned}
& \mathscr{S}_{\mathcal{H}_{\psi} B_{R}\left(a, s, r, \theta_{0}\right)} \\
= & a^{\frac{3}{4}} \int_{0}^{\infty} \int_{0}^{2 \pi} \hat{\psi}_{1}(a \rho \cos \theta) \hat{\psi}_{2}\left(a^{-\frac{1}{2}}(\tan \theta-s)\right) e^{2 \pi i \rho r \cos \left(\theta-\theta_{0}\right)} R^{2} \widehat{B}_{1}(R \rho) d \theta \rho d \rho \\
= & R^{2} a^{-\frac{5}{4}} \int_{0}^{\infty} \int_{0}^{2 \pi} \hat{\psi}_{1}(\rho \cos \theta) \hat{\psi}_{2}\left(a^{-\frac{1}{2}}(\tan \theta-s)\right) e^{2 \pi i \frac{\rho r}{a} \cos \left(\theta-\theta_{0}\right)} \widehat{B}_{1}\left(\frac{R \rho}{a}\right) d \theta \rho d \rho \\
= & R^{2} a^{-\frac{5}{4}} \int_{0}^{\infty} \eta\left(\rho, a, s, r, \theta_{0}\right) \widehat{B}_{1}\left(\frac{R \rho}{a}\right) \rho d \rho,
\end{aligned}
$$

where

$$
\begin{aligned}
\eta\left(\rho, a, s, r, \theta_{0}\right) & =\int_{0}^{2 \pi} \hat{\psi}_{1}(\rho \cos \theta) \hat{\psi}_{2}\left(a^{-\frac{1}{2}}(\tan \theta-s)\right) e^{2 \pi i \frac{\rho r}{a} \cos \left(\theta-\theta_{0}\right)} d \theta \\
& =\eta_{1}\left(\rho, a, s, r, \theta_{0}\right)-\eta_{2}\left(\rho, a, s, r, \theta_{0}\right)
\end{aligned}
$$

and 


$$
\begin{aligned}
& \eta_{1}\left(\rho, a, s, r, \theta_{0}\right)=\int_{-\frac{\pi}{2}}^{\frac{\pi}{2}} \hat{\psi}_{1}(\rho \cos \theta) \hat{\psi}_{2}\left(a^{-\frac{1}{2}}(\tan \theta-s)\right) e^{2 \pi i \frac{\rho r}{a} \cos \left(\theta-\theta_{0}\right)} d \theta \\
& \eta_{2}\left(\rho, a, s, r, \theta_{0}\right)=\int_{-\frac{\pi}{2}}^{\frac{\pi}{2}} \hat{\psi}_{1}(\rho \cos \theta) \hat{\psi}_{2}\left(a^{-\frac{1}{2}}(\tan \theta-s)\right) e^{-2 \pi i \frac{\rho r}{a} \cos \left(\theta-\theta_{0}\right)} d \theta
\end{aligned}
$$

In the last equality, we have used the fact that $\hat{\psi}_{1}$ is odd. Hence, using the asymptotic estimate for $J_{1}$, for small $a$ we have:

$$
\mathscr{S} \mathscr{H}_{\psi} B_{R}\left(a, s, r, \theta_{0}\right)=a^{\frac{1}{4}} \frac{R^{\frac{1}{2}}}{\pi}\left(I\left(a, s, r, \theta_{0}\right)+E\left(a, s, r, \theta_{0}\right)\right),
$$

where

$$
\begin{aligned}
I\left(a, s, r, \theta_{0}\right) & =\int_{0}^{\infty} \eta\left(\rho, a, s, r, \theta_{0}\right) \cos \left(\frac{2 \pi R \rho}{a}-\frac{3 \pi}{4}\right) \rho^{-\frac{1}{2}} d \rho, \\
E\left(a, s, r, \theta_{0}\right) & =\int_{0}^{\infty} \eta\left(\rho, a, s, r, \theta_{0}\right) O\left(\left(\frac{R \rho}{a}\right)^{-3 / 2}\right) \rho^{-\frac{1}{2}} d \rho .
\end{aligned}
$$

At this point, taking advantage of the assumptions on the support of $\hat{\psi}_{1}$ and $\hat{\psi}_{2}$, one can show that, as $a \rightarrow 0$, we have that

$$
a^{-\beta} E=O\left(a^{\frac{3}{2}-\beta}\right) \rightarrow 0
$$

uniformly for $s, r, R$, for each $\beta \in(0,1)$.

It follows that the asymptotic behaviour of $\mathscr{S} \mathscr{H}_{\psi} B_{R}\left(a, s, r, \theta_{0}\right)$, as $a \rightarrow 0$, is controlled by the integral $I\left(a, s, r, \theta_{0}\right)$. The rest of the proof is divided in several cases, depending on the values of $s$ and $r$, which we briefly summarize.

Case 1: $s \neq \tan \theta_{0}$ (non-normal orientation).

In this case, since the derivative of the phase of the exponential in the integrals (12) and (13) does not vanish, an integration by parts argument shows that $\left|\eta\left(\rho, a, s, r, \theta_{0}\right)\right| \leq C_{N} a^{N}$, for any $N>0$. This implies that $I\left(a, s, r, \theta_{0}\right)$ has rapid asymptotic decay, as $a \rightarrow 0$.

Case 2: $s=\tan \theta_{0}$, but $r \neq R$ (away from the boundary)

The original argument in [6] was rather involved. Lemma 2 below, which is used in the general result, provides a simpler proof for this situation.

Case 3: $s=\tan \theta_{0}, r=R$ (normal orientation)

Setting $s=\tan \theta_{0}$ and $t=R\left(\cos \theta_{0}, \sin \theta_{0}\right)$, a direct computation leads to the expression: 


$$
\begin{aligned}
\lim _{a \rightarrow 0} a^{-\frac{3}{4}}\left|\mathscr{S}_{\mathcal{H}_{\psi}} B_{R}(a, s, t)\right| & =\lim _{a \rightarrow 0} a^{-\frac{3}{4}}\left|\mathscr{S}_{\mathscr{H}_{\psi}} B_{R}\left(a, \tan \theta_{0}, R, \theta_{0}\right)\right| \\
& =\mid-\frac{e^{-\frac{3 \pi i}{4}}}{2} \int_{0}^{\infty} \hat{\psi}_{1}\left(\rho \cos \theta_{0}\right) \rho^{-\frac{1}{2}} \overline{h(\rho, R)} d \rho \\
& +\frac{e^{\frac{3 \pi i}{4}}}{2} \int_{0}^{\infty} \hat{\psi}_{1}\left(\rho \cos \theta_{0}\right) \rho^{-\frac{1}{2}} h(\rho, R) d \rho \mid
\end{aligned}
$$

where

$$
h(\rho, R)=\frac{R^{\frac{1}{2}}}{\pi} \int_{-1}^{1} \hat{\psi}_{2}\left(u \sec ^{2} \theta_{0}\right) e^{-\pi i \rho R u^{2}} d u .
$$

Combining (14) and (15) and using the fact that $\hat{\psi}_{2}$ is even, it follows that

$$
\begin{aligned}
& \lim _{a \rightarrow 0} a^{-\frac{3}{4}}\left|\mathscr{S} \mathscr{H}_{\psi} B_{R}\left(a, \tan \theta_{0}, R, \theta_{0}\right)\right| \\
= & \frac{\sqrt{2 R}}{\pi}\left|\int_{0}^{\infty} \hat{\psi}_{1}\left(\rho \cos \theta_{0}\right) \rho^{-\frac{1}{2}} \int_{0}^{1} \hat{\psi}_{2}\left(u \sec ^{2} \theta_{0}\right)\left(\sin \left(\pi \rho R u^{2}\right)+\cos \left(\pi \rho R u^{2}\right)\right) d u d \rho\right| .
\end{aligned}
$$

The proof is completed using the fact that $\hat{\psi}_{1}$ is decreasing and applying the following Lemma, which is also found in [7].

Lemma 1. Let $\psi_{2} \in L^{2}(\mathbb{R})$ be such that $\left\|\psi_{2}\right\|_{2}=1$, supp $\hat{\psi}_{2} \subset[-1,1]$ and $\hat{\psi}_{2}$ is an even function and is nonnegative and decreasing on $[0,1]$. Then, for each $\rho>0$, one has

$$
\int_{0}^{1} \hat{\psi}_{2}(u)\left(\sin \left(\pi \rho u^{2}\right)+\cos \left(\pi \rho u^{2}\right)\right) d u>0 .
$$

\subsection{General 2D boundaries}

As indicated above, the characterization result for the boundaries of $2 \mathrm{D}$ regions holds for general bounded planar regions $S \subset \mathbb{R}^{2}$ whose boundary is piecewise smooth.

More precisely, we assume that the boundary set of $S$, denoted by $\partial S$, is a simple curve, of finite length $L$, which is smooth except possibly for finitely many corner points. To precisely define the corner points, it is useful to introduce a parametrization for $\partial S$. Namely, let $\alpha(t)$ be the parametrization of $\partial S$ with respect to the arc length parameter $t$. For any $t_{0} \in(0, L)$ and any $j \geq 0$, we assume that $\lim _{t \rightarrow t_{0}^{-}} \alpha^{(j)}(t)=\alpha^{(j)}\left(t_{0}^{-}\right)$and $\lim _{t \rightarrow t_{0}^{+}} \alpha^{(j)}(t)=\alpha^{(j)}\left(t_{0}^{+}\right)$exist. Also, let $\mathbf{n}\left(t^{-}\right), \mathbf{n}\left(t^{+}\right)$be the outer normal direction(s) of $\partial S$ at $\alpha(t)$ from the left and right, respectively; if these two are equal, we write them as $\mathbf{n}(t)$. Similarly, for the curvature of $\partial S$, we use the notation $\kappa\left(t^{-}\right), \kappa\left(t^{+}\right)$and $\kappa(t)$.

We say that $p=\alpha\left(t_{0}\right)$ is a corner point of $\partial S$ if either $(i) \alpha^{\prime}\left(t_{0}^{-}\right) \neq \pm \alpha^{\prime}\left(t_{0}^{+}\right)$or (ii) $\alpha^{\prime}\left(t_{0}^{-}\right)= \pm \alpha^{\prime}\left(t_{0}^{+}\right)$, but $\kappa\left(t_{0}^{-}\right) \neq \kappa\left(t_{0}^{+}\right)$. When (i) holds, we say that $p$ is a corner point of first type, while when (ii) holds, we say that $p$ is a corner point of second 
type. On the other hand, if $\alpha(t)$ is infinitely many times differentiable at $t_{0}$, we say that $\alpha\left(t_{0}\right)$ is a regular point of $\partial S$. Finally, we say that the boundary curve $\alpha(t)$ is piecewise smooth if the values $\alpha(t)$ are regular points for all $0 \leq t \leq L$, except for finitely many corner points.

Notice that it is possible to relax the assumptions on the regularity, by assuming that the regular points of $\partial S$ are $M$ times differentiable, for $M \in \mathbb{N}$, rather than infinitely differentiable. All the results presented below can be adapted to the case of piecewise $C^{M}$ boundary curves, for $M \geq 3$.

Let $p=\alpha\left(t_{0}\right)$ be a regular point and let $s=\tan \left(\theta_{0}\right)$ with $\theta_{0} \in\left(-\frac{\pi}{2}, \frac{\pi}{2}\right)$. Let $\Theta\left(\theta_{0}\right)=\left(\cos \theta_{0}, \sin \theta_{0}\right)$. We say that $s$ corresponds to the normal direction of $\partial S$ at $p$ if $\Theta\left(\theta_{0}\right)= \pm \mathbf{n}\left(t_{0}\right)$. We can proceed similarly when $\alpha\left(t_{0}\right)$ is a corner point. In this case, however, there may be two outer normal directions $\mathbf{n}\left(t_{0}^{-}\right)$and $\mathbf{n}\left(t_{0}^{+}\right)$.

We are now ready to state the following results from [7].

Theorem 2. Let $B=\chi_{S}$, where $S \subset \mathbb{R}^{2}$ satisfies the properties described above.

(i) If $p \notin \partial S$ then

$$
\lim _{a \rightarrow 0^{+}} a^{-N} \mathscr{S}_{H_{\psi}} B\left(a, s_{0}, p\right)=0, \quad \text { for all } N>0 .
$$

(ii) If $p \in \partial S$ is a regular point and $s=s_{0}$ does not correspond to the normal direction of $\partial S$ at $p$ then

$$
\lim _{a \rightarrow 0^{+}} a^{-N} \mathscr{S} \mathscr{H}_{\psi} B\left(a, s_{0}, p\right)=0, \quad \text { for all } N>0 .
$$

(iii) If $p \in \partial S$ is a regular point and $s=s_{0}$ corresponds to the normal direction of $\partial S$ at $p$ then

$$
\lim _{a \rightarrow 0^{+}} a^{-\frac{3}{4}} \mathscr{S}_{\mathscr{H}}^{\psi} B\left(a, s_{0}, p\right) \neq 0 .
$$

In the case where $p \in \partial S$ is a corner point we have the following result.

Theorem 3. Let $B=\chi_{S}$, where $S \subset \mathbb{R}^{2}$ satisfies the properties described above.

(i) If $p$ is a corner point of the first type and $s=s_{0}$ does not correspond to any of the normal directions of $\partial S$ at $p$, then

$$
\lim _{a \rightarrow 0^{+}} a^{-\frac{9}{4}} \mathscr{S} \mathscr{H} \psi\left(a, s_{0}, p\right)<\infty .
$$

(ii) If $p$ is a corner point of the second type and $s=s_{0}$ does not correspond to any of the normal directions of $\partial S$ at $p$, then

$$
\lim _{a \rightarrow 0^{+}} a^{-\frac{9}{4}} \mathscr{S}_{\mathscr{H}}^{\psi} B\left(a, s_{0}, p\right) \neq 0
$$

(iii) If $s=s_{0}$ corresponds to one of the normal directions of $\partial S$ at $p$ then

$$
\lim _{a \rightarrow 0^{+}} a^{-\frac{3}{4}} \mathscr{S}_{H_{\psi}} B\left(a, s_{0}, p\right) \neq 0
$$


Theorem 2 shows that, if $p \in \partial S$ is a regular point, the continuous shearlet transform decays rapidly, asymptotically for $a \rightarrow 0$, unless $s=s_{0}$ corresponds to the normal direction of $\partial S$ at $p$, in which case

$$
\mathscr{S} \mathscr{H}_{\psi} B\left(a, s_{0}, p\right) \sim O\left(a^{\frac{3}{4}}\right), \text { as } a \rightarrow 0 .
$$

This result contains the situation where $B$ is a disk, as a special case.

Theorem 3 shows that, at a corner points $p$, the asymptotic decay of the continuous shearlet transform depends both on the tangent and the curvature at $p$. If $s=s_{0}$ corresponds to one of the normal directions of $\partial S$ at $p$, then the continuous shearlet transform decays as

$$
\mathscr{S} \mathscr{H}_{\psi} B\left(a, s_{0}, p\right) \sim O\left(a^{\frac{3}{4}}\right), \text { as } a \rightarrow 0
$$

This is the same decay rate as for regular points, when $s_{0}$ corresponds to the normal direction (but now there are two normal directions). If $p$ is a corner point of the second type and $s$ does not correspond to any of the normal directions, then

$$
\mathscr{S} \mathscr{H}_{\psi} B\left(a, s_{0}, p\right) \sim O\left(a^{\frac{9}{4}}\right), \text { as } a \rightarrow 0,
$$

which is a faster decay rate than in the normal-orientation case. Finally, if $p$ is a corner point of the first type and $s_{0}$ does not correspond to any of the normal directions, then, by the theorem, we only know that the asymptotic decay of $\left|\mathscr{S}_{\mathscr{H}} \boldsymbol{B}\left(a, s_{0}, p\right)\right|$ is not slower than $O\left(a^{\frac{9}{4}}\right)$; however the decay could be faster than $O\left(a^{\frac{9}{4}}\right)$. For example, as shown in [7], if $p$ is a corner point of a half disk, when $s_{0}$ does not correspond to the normal directions, we have that

$$
\mathscr{S} \mathscr{H}_{\psi} B\left(a, s_{0}, p\right) \sim O\left(a^{\frac{9}{4}}\right), \text { as } a \rightarrow 0 .
$$

However, when $p$ is corner point of a polygon $S$ and $s_{0}$ does not correspond to the normal directions, for any $N \in \mathbb{N}$, there is a constant $C_{N}>0$ such that

$$
\left|\mathscr{S} \mathscr{H}_{\psi} B\left(a, s_{0}, p\right)\right| \leq C_{N} a^{N}, \text { as } a \rightarrow 0 .
$$

\subsection{Proofs of Theorems 2 and 3}

It is clear that the proof used for the disk in Section 2.1 cannot be extended to this case directly since that argument requires an explicit expression of the Fourier transform of the region $B$. Instead, using the divergence theorem, we can express the Fourier transform of our general region $B \subset \mathbb{R}^{2}$ as 


$$
\begin{aligned}
\hat{B}(\xi) & =\widehat{\chi}_{S}(\xi)=-\frac{1}{2 \pi i|\xi|} \int_{\partial S} e^{-2 \pi i\langle\xi, x\rangle} \Theta(\theta) \cdot \mathbf{n}(x) d \sigma(x) \\
& =-\frac{1}{2 \pi i \rho} \int_{0}^{L} e^{-2 \pi i \rho \Theta(\theta) \cdot \alpha(t)} \Theta(\theta) \cdot \mathbf{n}(t) d t
\end{aligned}
$$

where $\xi=\rho \Theta(\theta), \Theta(\theta)=(\cos \theta, \sin \theta)$. Notice that this idea for representing the Fourier transform of the characteristic function of a bounded region is used, for example, in [13].

Hence, using (22), we have that

$$
\begin{aligned}
& \mathscr{S}_{\mathscr{H}_{\psi}} B(a, s, p) \\
& =\left\langle B, \psi_{a, s, p}\right\rangle \\
& =\int_{0}^{2 \pi} \int_{0}^{\infty} \hat{B}(\rho, \theta) \overline{\hat{\psi}_{a, s, p}^{(d)}}(\rho, \theta) \rho d \rho d \theta \\
& =-\frac{1}{2 \pi i} \int_{0}^{2 \pi} \int_{0}^{\infty} \int_{0}^{L} \overline{\hat{\psi}_{a, s, p}^{(d)}}(\rho, \theta) e^{-2 \pi i \rho \Theta(\theta) \cdot \alpha(t)} \Theta(\theta) \cdot \mathbf{n}(t) d t d \rho d \theta,
\end{aligned}
$$

where the upper-script in $\psi_{a, s, p}^{(d)}$ is either $d=h$, when $|s| \leq 1$, or $d=v$, when $|s|>1$.

The first useful observation is that the asymptotic decay of the shearlet transform $\mathscr{S} \mathscr{H}_{\psi} B(a, s, p)$, as $a \rightarrow 0$, is only determined by the values of the boundary $\partial S$ which are "close" to $p$. To state this fact, for $\varepsilon>0$, let $D(\varepsilon, p)$ be the ball in $\mathbb{R}^{2}$ of radius $\varepsilon$ and center $p$, and $D^{c}(\varepsilon, p)=\mathbb{R}^{2} \backslash D(\varepsilon, p)$. Hence, using (23), we can write the shearlet transform of $B$ as

$$
\mathscr{S} \mathscr{H}_{\psi} B(a, s, p)=I_{1}(a, s, p)+I_{2}(a, s, p),
$$

where

$$
\begin{aligned}
& I_{1}(a, s, p) \\
& =-\frac{1}{2 \pi i} \int_{0}^{2 \pi} \int_{0}^{\infty} \int_{\partial S \cap D(\varepsilon, p)} \overline{\hat{\psi}_{a, s, p}^{(d)}}(\rho, \theta) e^{-2 \pi i \rho \Theta(\theta) \cdot \alpha(t)} \Theta(\theta) \cdot \mathbf{n}(t) d t d \rho d \theta, \\
& I_{2}(a, s, p) \\
& =-\frac{1}{2 \pi i} \int_{0}^{2 \pi} \int_{0}^{\infty} \int_{\partial S \cap D^{c}(\varepsilon, p)} \overline{\hat{\psi}_{a, s, p}^{(d)}}(\rho, \theta) e^{-2 \pi i \rho \Theta(\theta) \cdot \alpha(t)} \Theta(\theta) \cdot \mathbf{n}(t) d t d \rho d \theta .
\end{aligned}
$$

Thus, the following Localization Lemma shows that $I_{2}$ has rapid asymptotic decay, at fine scales.

Lemma 2 (Localization Lemma). Let $I_{2}(a, s, p)$ be given by (25). For any positive integer $N$, there is a constant $C_{N}>0$ such that

$$
\left|I_{2}(a, s, p)\right| \leq C_{N} a^{\frac{N}{2}}
$$

asymptotically as $a \rightarrow 0$, uniformly for all $s \in \mathbb{R}$. 
Proof. We will only examine the behaviour of $I_{2}(a, s, p)$ for $|s| \leq 1$ (in which case we use the 'horizontal' shearlet transform). The case where $|s|>1$ is similar. We have:

$$
\begin{aligned}
I_{2}(a, s, p)= & -\frac{1}{2 \pi i} \int_{\partial S \cap D^{c}(\varepsilon, p)} \int_{0}^{2 \pi} \int_{0}^{\infty} \overline{\hat{\psi}_{a, s, p}^{(h)}}(\rho, \theta) e^{-2 \pi i \rho \Theta(\theta) \cdot \alpha(t)} \Theta(\theta) \cdot \mathbf{n}(t) d t d \rho d \theta \\
= & \frac{-a^{3 / 4}}{2 \pi i} \int_{\partial S \cap D^{c}(\varepsilon, p)} \int_{0}^{2 \pi} \int_{0}^{\infty} \hat{\psi}_{1}(a \rho \cos \theta) \hat{\psi}_{2}\left(a^{-1 / 2}(\tan \theta-s)\right) \\
& \times e^{2 \pi i \rho \Theta(\theta) \cdot p} d \rho d \theta e^{-2 \pi i \rho \Theta(\theta) \cdot \alpha(t)} \Theta(\theta) \cdot \mathbf{n}(t) d t \\
= & \frac{-a^{-1 / 4}}{2 \pi i} \int_{\partial S \cap D^{c}(\varepsilon, p)} \int_{0}^{2 \pi} \int_{0}^{\infty} \hat{\psi}_{1}(\rho \cos \theta) \hat{\psi}_{2}\left(a^{-1 / 2}(\tan \theta-s)\right) \\
& \times e^{2 \pi i \frac{\rho}{a} \Theta(\theta) \cdot(p-\alpha(t))} \Theta(\theta) \cdot \mathbf{n}(t) d \rho d \theta d t .
\end{aligned}
$$

By assumption, $\|p-\alpha(t)\| \geq \varepsilon$ for all $\alpha(t) \in \partial S \cap D^{c}(\varepsilon, p)$. Hence, there is a constant $C_{p}$ such that $\inf _{x \in \partial S \cap D^{c}(\varepsilon, p)}|p-x|=C_{p}$. Let $\mathscr{I}=\{\theta:|\tan \theta-s| \leq$ $\left.a^{\frac{1}{2}}\right\}, \mathscr{I}_{1}=\left\{\theta:|\Theta(\theta) \cdot(p-x)| \geq \frac{C_{p}}{\sqrt{2}}\right\} \cap \mathscr{I}$, and $\mathscr{I}_{2}=\mathscr{I} \backslash \mathscr{I}_{1}$. Since the vectors $\Theta(\theta), \Theta^{\prime}(\theta)$ form an orthonormal basis in $\mathbb{R}^{2}$, it follows that, on the set $\mathscr{I}_{2}$, we have $\left|\Theta^{\prime}(\theta) \cdot(p-x)\right| \geq \frac{C_{p}}{\sqrt{2}}$. Hence we can express the integrals $I_{2}$ as a sum of a term where $\theta \in \mathscr{I}_{1}$ and another term where $\theta \in \mathscr{I}_{2}$, and integrate by parts as follows. On $\mathscr{I}_{1}$, we integrate by parts with respect to the variable $\rho$; on $\mathscr{I}_{2}$ we integrate by parts with respect to the variable $\theta$. Doing this repeatedly, it yields that, for any positive integer $N,\left|I_{2}(a, s, p)\right| \leq C_{N} a^{\frac{N}{2}}$, uniformly in $s$. This finishes the proof of the lemma.

Let $\alpha(t)$ be the boundary curve $\partial S$, with $0 \leq t \leq L$. We may assume that $L>1$ and $p=(0,0)=\alpha(1)$. When $p$ is a corner point of $\partial S$, we can write

$$
\mathscr{C}=\partial S \cap D(\varepsilon,(0,0))=\mathscr{C}^{-} \cup \mathscr{C}^{+}
$$

where

$$
\mathscr{C}^{-}=\{\alpha(t): 1-\varepsilon \leq t \leq 1\}, \quad \mathscr{C}^{+}=\{\alpha(t): 1 \leq t \leq 1+\varepsilon\}
$$

When $p$ is a corner point of $\partial S$ and $s$ corresponds to one of the normal directions of $\partial S$ at $p$, it will be useful to replace the portion of $\partial S$ near $p$ by the Taylor polynomials of degree 2 on both sides of $p$. Notice that these two polynomials are not necessarily the same since $p$ is a corner point.

On the other hand, for regular point $p \in \partial S$, rather than using the arclength representation, we let $\mathscr{C}^{+}=\left\{\left(G^{+}(u), u\right), 0 \leq u \leq \varepsilon\right\}$ and $\mathscr{C}^{-}=\left\{\left(G^{-}(u), u\right),-\varepsilon \leq\right.$ $u \leq 0\}$, where $G^{+}(u)$ and $G^{-}(u)$ are smooth functions on $[0, \varepsilon]$ and $[-\varepsilon, 0]$, respectively. Without loss of generality, we may assume that $p=(0,0)$ so that $u_{0}=0$ and $G(0)=0$. Hence we define the quadratic approximation of $S$ near $p=(0,0)$ by $\partial S_{0}=\left(G_{0}(u), u\right)$, where $G_{0}$ is the Taylor polynomial of degree 2 of $G$ centered at the origin, given by $G_{0}(u)=G^{\prime}(0) u+\frac{1}{2} G^{\prime \prime}(0) u^{2}$. Hence we define $B_{0}=\chi_{S_{0}}$, where 
$S_{0}$ is obtained by replacing the curve $\partial S$ in $B=\chi_{S}$ with the quadratic curve $\partial S_{0}$ near the point $p=(0,0)$. If $p$ is a corner point, then $S_{0}$ is obtained by replacing the curve $\partial S$ near $p$ in $B=\chi_{S}$ with both the left and right quadratic curves $\partial S_{0}$ near the point $p$. For simplicity, we only prove the following result for a regular point $p$. The argument for a corner point $p$ is similar.

Lemma 3. For any $|s| \leq \frac{3}{2}$, we have

$$
\left.\lim _{a \rightarrow 0^{+}} a^{-\frac{3}{4}} \mid \mathscr{S}_{\mathscr{H}}^{\psi} B(a, s, 0)\right)-\mathscr{S} \mathscr{H}_{\psi} B_{0}(a, s, 0) \mid=0 .
$$

Proof. Notice that, since we assume $|s| \leq \frac{3}{2}$, we use the system of 'horizontal' shearlets only.

Let $\gamma$ be chosen such that $\frac{3}{8}<\gamma<\frac{1}{2}$ and assume that $a$ is sufficiently small, so that $a^{\gamma}<\varepsilon$. A direct calculation shows that

$$
\begin{aligned}
\left|\mathscr{S}_{\mathscr{H}_{\psi}} B(a, s, 0)-\mathscr{S} \mathscr{H}_{\psi} B_{0}(a, s, 0)\right| & \leq \int_{\mathbb{R}^{2}}\left|\psi_{a, s, 0}^{(h)}(x)\right|\left|\chi_{S}(x)-\chi_{S_{0}}(x)\right| d x \\
& =T_{1}(a)+T_{2}(a),
\end{aligned}
$$

where $x=\left(x_{1}, x_{2}\right) \in \mathbb{R}^{2}$ and

$$
\begin{aligned}
& T_{1}(a)=a^{-\frac{3}{4}} \int_{D(a \gamma,(0,0))}\left|\psi^{(h)}\left(M_{a s}^{-1} x\right)\right|\left|\chi_{S}(x)-\chi_{S_{0}}(x)\right| d x, \\
& T_{2}(a)=a^{-\frac{3}{4}} \int_{D^{c}\left(a^{\gamma},(0,0)\right)}\left|\psi^{(h)}\left(M_{a s}^{-1} x\right)\right|\left|\chi_{S}(x)-\chi_{S_{0}}(x)\right| d x .
\end{aligned}
$$

Observe that:

$$
T_{1}(a) \leq C a^{-\frac{3}{4}} \int_{D\left(a^{\gamma},(0,0)\right)}\left|\chi_{S}(x)-\chi_{S_{0}}(x)\right| d x
$$

To estimate the above quantity, it is enough to compute the area between the regions $S$ and $S_{0}$. Since $G_{0}$ is the Taylor polynomial of $G$ of degree 2, we have

$$
T_{1}(a) \leq C a^{-\frac{3}{4}} \int_{|x|<a^{\gamma}}|x|^{3} d x \leq C a^{4 \gamma-\frac{3}{4}} .
$$

Since $\gamma>\frac{3}{8}$, the above estimate shows that $T_{1}(a)=o\left(a^{\frac{3}{4}}\right)$.

The assumptions on the generator function $\psi^{(h)}$ of the shearlet system $\psi^{(h)}$ imply that, for each $N>0$, there is a constant $C_{N}>0$ such that $|\psi(x)| \leq C_{N}\left(1+|x|^{2}\right)^{-N}$. It is easy to see that $\left(M_{a s}\right)^{-1}=A_{a} B_{s}$, where $B_{s}=\left(\begin{array}{ll}1 & s \\ 0 & 1\end{array}\right)$ and $A_{a}=\left(\begin{array}{cc}a^{-1} & 0 \\ 0 & a^{-\frac{1}{2}}\end{array}\right)$. Also, it is easy to verify that, for all $|s| \leq \frac{3}{2}$, there is a constant $C_{0}>0$ such that $\left\|B_{s} x\right\|^{2} \geq C_{0}\|x\|^{2}$, or $\left(x_{1}+s x_{2}\right)^{2}+x_{2}^{2} \geq C_{0}\left(x_{1}^{2}+x_{2}^{2}\right)$ for all $x \in \mathbb{R}^{2}$. Thus, for $a<1$, we can estimate $T_{2}(a)$ as: 


$$
\begin{aligned}
T_{2}(a) & \leq C a^{-\frac{3}{4}} \int_{D^{c}\left(a^{\gamma},(0,0)\right)}\left|\psi^{(h)}\left(M_{a s} x\right)\right| d x \\
& \leq C_{N} a^{-\frac{3}{4}} \int_{D^{c}\left(a^{\gamma},(0,0)\right)}\left(1+\left(a^{-1}\left(x_{1}+s x_{2}\right)\right)^{2}+\left(a^{-\frac{1}{2}} x_{2}\right)^{2}\right)^{-N} d x \\
& \leq C_{N} a^{-\frac{3}{4}} \int_{D^{c}\left(a^{\gamma},(0,0)\right)}\left(\left(a^{-1 / 2}\left(x_{1}+s x_{2}\right)\right)^{2}+\left(a^{-\frac{1}{2}} x_{2}\right)^{2}\right)^{-N} d x \\
& =C_{N} a^{N-\frac{3}{4}} \int_{D^{c}\left(a^{\gamma},(0,0)\right)}\left(x_{1}^{2}+x_{2}^{2}\right)^{-N} d x \\
& =C_{N} a^{N-\frac{3}{4}} \int_{a^{\gamma}}^{\infty} r^{1-2 N} d r \\
& =C_{N} a^{2 N\left(\frac{1}{2}-\gamma\right)} a^{2 \gamma-\frac{3}{4}},
\end{aligned}
$$

where the constant $C_{0}$ was absorbed in the constant $C_{N}$.

Since $\gamma<\frac{1}{2}$ and $N$ can be chosen arbitrarily large, it follows that $T_{2}(a)=o\left(a^{\frac{3}{4}}\right)$.

We can now proceed with the proof of Theorem 2. Here and in the proof of Theorem 3, it will be sufficient to examine the case of the horizontal shearlets only. The case of the vertical ones can be handled in essentially the same way.

\section{Proof of Theorem 2.}

- Part (i) This follows directly from Lemma 2.

- Part (ii) Assume that $s=s_{0}$ does not correspond to any of the normal directions of $\partial S$ at $p=(0,0)$. We write $s_{0}=\tan \theta_{0}$, where we assume that $\left|\theta_{0}\right| \leq \frac{\pi}{4}$. Otherwise, for the case $\frac{\pi}{4}<\left|\theta_{0}\right| \leq \frac{\pi}{2}$, one will use the "vertical" shearlet transform, and the argument is very similar to the one we will present below. Hence, we have that

$I_{1}\left(a, s_{0}, 0\right)=-\frac{a^{-\frac{1}{4}}}{2 \pi i} \int_{0}^{\infty} \int_{0}^{2 \pi} \hat{\psi}_{1}(\rho \cos \theta) \hat{\psi}_{2}\left(a^{-1 / 2}\left(\tan \theta-\tan \theta_{0}\right)\right) K(a, \rho, \theta) d \theta d \rho$

where

$$
K(a, \rho, \theta)=\int_{1-\varepsilon}^{1+\varepsilon} e^{-2 \pi i \frac{\rho}{a} \Theta(\theta) \cdot \alpha(t)} \Theta(\theta) \cdot \mathbf{n}(t) d t
$$

Letting $G(t) \in C_{0}^{\infty}(\mathbb{R})$ with $G(t)=1$ for $|t-1| \leq \frac{\varepsilon}{4}$ and $G(t)=0$ for $|t-1|>\frac{3 \varepsilon}{4}$, we write

$$
I_{1}\left(a, s_{0}, 0\right)=I_{11}\left(a, s_{0}, 0\right)+I_{12}\left(a, s_{0}, 0\right)
$$

where

$$
\begin{aligned}
& I_{11}\left(a, s_{0}, 0\right)=-\frac{a^{-\frac{1}{4}}}{2 \pi i} \int_{0}^{\infty} \int_{0}^{2 \pi} \hat{\psi}_{1}(\rho \cos \theta) \hat{\psi}_{2}\left(a^{-\frac{1}{2}}\left(\tan \theta-\tan \theta_{0}\right)\right) K_{1}(a, \rho, \theta) d \theta d \rho \\
& I_{12}\left(a, s_{0}, 0\right)=-\frac{a^{-\frac{1}{4}}}{2 \pi i} \int_{0}^{\infty} \int_{0}^{2 \pi} \hat{\psi}_{1}(\rho \cos \theta) \hat{\psi}_{2}\left(a^{-\frac{1}{2}}\left(\tan \theta-\tan \theta_{0}\right)\right) K_{2}(a, \rho, \theta) d \theta d \rho
\end{aligned}
$$


and

$$
\begin{aligned}
& K_{1}(a, \rho, \theta)=\int_{1-\varepsilon}^{1+\varepsilon} e^{-2 \pi i \frac{\rho}{a} \Theta(\theta) \cdot \alpha(t)} \Theta(\theta) \cdot \mathbf{n}(t) G(t) d t \\
& K_{2}(a, \rho, \theta)=\int_{1-\varepsilon}^{1+\varepsilon} e^{-2 \pi i \frac{\rho}{a} \Theta(\theta) \cdot \alpha(t)} \Theta(\theta) \cdot \mathbf{n}(t)(1-G(t)) d t .
\end{aligned}
$$

From the definition of $G(t)$, we have $1-G(t)=0$ for $|t-1| \leq \frac{\varepsilon}{4}$. Since the boundary curve $\{\alpha(t), 0 \leq t \leq L\}$ is simple and $p=(0,0)=\alpha(1)$, it follows that there exists a $c_{0}>0$ such that $\|\alpha(t)\| \geq c_{0}$ for all $t$ with $\frac{\varepsilon}{4} \leq|t-1| \leq \varepsilon$. Replacing the set $D^{c}(\varepsilon, p)$ by the set $\left\{\alpha(t), \frac{\varepsilon}{4} \leq|t-1| \leq \varepsilon\right\}$, one can repeat the argument of Lemma 2 for $I_{12}\left(a, s_{0}, 0\right)$ to show that $\left|I_{12}\left(a, s_{0}, 0\right)\right| \leq C_{N} a^{N}$ for any $N>0$.

Recall that when $a \rightarrow 0$, we have $\theta \rightarrow \theta_{0}$. Since $s_{0}$ does not correspond to the normal direction at $p$, one can choose $\varepsilon$ sufficient small so that $\Theta(\theta) \cdot \alpha^{\prime}(t) \neq 0$ for $|t-1| \leq \varepsilon$ and for all small $a$ (and hence for $\theta$ near $\theta_{0}$ ). Also from the assumption on $G(t)$, it follows that $G^{(n)}(1-\varepsilon)=0$ and $G^{(n)}(1+\varepsilon)=0$ for all $n \geq 0$. Writing

$$
e^{-2 \pi i \frac{\rho}{a} \Theta(\theta) \cdot \alpha(t)}=\frac{-a}{2 \pi i \rho \Theta(\theta) \cdot \alpha^{\prime}(t)}\left(e^{-2 \pi i \frac{\rho}{a} \Theta(\theta) \cdot \alpha(t)}\right)^{\prime}
$$

it follows that

$$
\begin{aligned}
K_{1}(a, \rho, \theta) & =-\frac{a}{2 \pi i \rho} \int_{1-\varepsilon}^{1+\varepsilon}\left(e^{-2 \pi i \frac{\rho}{a} \Theta(\theta) \cdot \alpha(t)}\right)^{\prime} \frac{\Theta(\theta) \cdot \mathbf{n}(t)}{\Theta(\theta) \cdot \alpha^{\prime}(t)} G(t) d t \\
& =\frac{a i}{2 \pi \rho}\left\{\left(e^{-2 \pi i \frac{\rho}{a} \Theta(\theta) \cdot \alpha(t)} \frac{\Theta(\theta) \cdot \mathbf{n}(t)}{\Theta(\theta) \cdot \alpha^{\prime}(t)} G(t)\right)_{1-\varepsilon}^{1+\varepsilon}+K_{3}(a, \rho, \theta)\right\} \\
& =\frac{a i}{2 \pi \rho} K_{3}(a, \rho, \theta),
\end{aligned}
$$

where we used the fact that $G(1-\varepsilon)=0, G(1+\varepsilon)=0$.

Repeating the above argument for $K_{3}(a, \rho, \theta)$ and using induction, it follows that for all $N>0$ there exists a $C_{N}>0$ such that $\left|K_{1}(a, \rho, \theta)\right| \leq C_{N} a^{N}$ and, hence, that $\left|I_{11}\left(a, s_{0}, 0\right)\right| \leq C_{N} a^{N}$.

- Part (iii) We may assume that $p=(0,0)$ and $s=\tan \theta_{0}$ for some $\left|\theta_{0}\right| \leq \frac{\pi}{4}$. Let $S, G, S_{0}, G_{0}$ be defined as in Lemma 3 . Thus, according to Lemma 3 , on the curve $\mathscr{C}$, one can use $G_{0}(u)$ to replace $G^{+}(u)$ since the approximation error is $o\left(a^{\frac{3}{4}}\right)$. For simplicity of notation, in the following we will use $G$ to denote $G^{+}$.

Using polar coordinates, we can express $I_{1}(a, 0,0)$, evaluated on $S_{0}$, as

$$
\begin{aligned}
I_{1}(a, 0,0) & =-\frac{1}{2 \pi i a^{\frac{1}{4}}} \int_{0}^{\infty} \int_{0}^{2 \pi} \hat{\psi}_{1}(\rho \cos \theta) \hat{\psi}_{2}\left(a^{-\frac{1}{2}}\left(\tan \theta-\tan \theta_{0}\right)\right) \\
& \times \int_{-\varepsilon}^{\varepsilon} e^{-2 \pi i \frac{\rho}{a}\left(\cos \theta G_{0}(u)+\sin \theta u\right)}\left(-\cos \theta+\sin \theta G_{0}^{\prime}(u)\right) d u d \theta d \rho .
\end{aligned}
$$

By Lemma 2 and Lemma 3, to complete the proof it is sufficient to show 


$$
\lim _{a \rightarrow 0^{+}} a^{-\frac{3}{4}} I_{1}(a, 0,0) \neq 0 .
$$

Let $H_{\theta}(u)=\cos \theta G_{0}(u)+u \sin \theta$ and $A=\frac{1}{2} G^{\prime \prime}(0)$. Since $s=\tan \theta_{0}$ corresponds to the normal direction of $S_{0}$ at $p=(0,0)$, it follows that $H_{\theta_{0}}^{\prime}(0)=0$. This implies that $G^{\prime}(0)=-\tan \theta_{0}$, so that $H_{\theta}(u)=\cos \theta\left(-u \tan \theta_{0}+A u^{2}\right)+u \sin \theta=A u^{2} \cos \theta+$ $u\left(\sin \theta-\cos \theta \tan \theta_{0}\right)$. We will consider separately the cases $A \neq 0$ and $A=0$.

Case 1: $A \neq 0$. In this case, we may assume that $A>0$ since the case $A<0$ is similar. We have that

$$
\begin{aligned}
& \int_{-\varepsilon}^{\varepsilon} e^{-2 \pi i \frac{\rho}{a}\left(G_{0}(u) \cos \theta+u \sin \theta\right)}\left(-\cos \theta+G_{0}^{\prime}(u) \sin \theta\right) d u \\
= & e^{\frac{\rho \pi i}{a} \frac{\left(\sin \theta-\cos \theta \tan \theta_{0}\right)^{2}}{2 A \cos \theta} \int_{-\varepsilon}^{\varepsilon} e^{-2 \pi i \frac{\rho}{a} A\left(u-u_{\theta}\right)^{2} \cos \theta}\left(2 A u \sin \theta-\cos \theta-\sin \theta \tan \theta_{0}\right) d u} \\
= & K_{0}(\theta, a)+K_{1}(\theta, a),
\end{aligned}
$$

where $u_{\theta}=-\frac{\sin \theta-\cos \theta \tan \theta_{0}}{2 A \cos \theta}=-\frac{1}{2 A}\left(\tan \theta-\tan \theta_{0}\right)$,

$$
\begin{aligned}
& K_{0}(\theta, a)=-\left(\cos \theta+\sin \theta \tan \theta_{0}\right) e^{\frac{\rho \pi i}{2 A} \frac{\left(\tan \theta-\tan \theta_{0}\right)^{2}}{a}} \int_{-\varepsilon}^{\varepsilon} e^{-2 \pi i \frac{\rho}{a} \cos \theta A\left(u-u_{\theta}\right)^{2}} d u, \\
& K_{1}(\theta, a)=2 A \sin \theta e^{\frac{\rho \pi i}{2 A} \frac{\left(\tan \theta-\tan \theta_{0}\right)^{2}}{a}} \int_{-\varepsilon}^{\varepsilon} e^{-2 \pi i \frac{\rho}{a} \cos \theta A\left(u-u_{\theta}\right)^{2}} u d u .
\end{aligned}
$$

In the expression of $I_{1}$, the interval $[0,2 \pi]$ of the integral in $\theta$ can be broken into the subintervals $\left[-\frac{\pi}{2}, \frac{\pi}{2}\right]$ and $\left[\frac{\pi}{2}, \frac{3 \pi}{2}\right]$. On $\left[\frac{\pi}{2}, \frac{3 \pi}{2}\right]$, we let $\theta^{\prime}=\theta-\pi$ so that $\theta^{\prime} \in$ $\left[-\frac{\pi}{2}, \frac{\pi}{2}\right]$ and that $\sin \theta=-\sin \theta^{\prime}, \cos \theta=-\cos \theta^{\prime}$. Using this observation and the fact that $\hat{\psi}_{1}$ is an odd function, it follows that $I_{1}(a, 0,0)=I_{10}(a, 0,0)+I_{11}(a, 0,0)$, where for $j=0,1$,

$$
\begin{aligned}
I_{1 j}(a, 0,0) & =-\frac{1}{2 \pi i a^{\frac{1}{4}}} \int_{0}^{\infty} \int_{-\frac{\pi}{2}}^{\frac{\pi}{2}} \hat{\psi}_{1}(\rho \cos \theta) \hat{\psi}_{2}\left(a^{-\frac{1}{2}}(\tan \theta)\right) K_{j}(\theta, a) d \theta d \rho . \\
& +\frac{1}{2 \pi i a^{\frac{1}{4}}} \int_{0}^{\infty} \int_{-\frac{\pi}{2}}^{\frac{\pi}{2}} \hat{\psi}_{1}(\rho \cos \theta) \hat{\psi}_{2}\left(a^{-\frac{1}{2}}(\tan \theta)\right) K_{j}(\theta+\pi, a) d \theta d \rho .
\end{aligned}
$$

For $\theta \in\left(-\frac{\pi}{2}, \frac{\pi}{2}\right)$, let $t=a^{-\frac{1}{2}}\left(\tan \theta-\tan \theta_{0}\right)$ and $a^{-\frac{1}{2}} u=u^{\prime}$. Since $a \rightarrow 0$ implies $\theta \rightarrow \theta_{0}$, we obtain:

$$
\begin{aligned}
\lim _{a \rightarrow 0^{+}} a^{-\frac{1}{2}} K_{0}(\theta, a) & =-\sec \theta_{0} e^{\frac{i \pi \rho}{2 A} t^{2}} \int_{-\infty}^{\infty} e^{-2 \pi i \rho A \cos \theta_{0}\left(u-\frac{t}{2 A}\right)^{2}} d u \\
& =-\sec \theta_{0} e^{\frac{i \pi \rho}{2 A} t^{2}} \int_{-\infty}^{\infty} e^{-2 \pi i \rho A \cos \theta_{0} u^{2}} d u
\end{aligned}
$$

Similarly, we have that

$$
\lim _{a \rightarrow 0^{+}} a^{-\frac{1}{2}} K_{0}(\theta+\pi, a)=\sec \theta_{0} e^{-\frac{i \pi \rho}{2 A} t^{2}} \int_{-\infty}^{\infty} e^{2 \pi i \rho A \cos \theta_{0} u^{2}} d u .
$$


Using the calculations of $K_{0}(\theta, a)$ and $K_{0}(\theta+\pi, a)$, it follows from the additional factor $u$ inside the integral of $K_{1}$ that $K_{1}(\theta, a)=O(a)$ and $K_{1}(\theta+\pi, a)=O(a)$. Thus, we obtain:

$$
\lim _{a \rightarrow 0^{+}} \frac{2 \pi i}{a^{3 / 4}} I_{1}(a, 0,0)=\lim _{a \rightarrow 0^{+}} \frac{2 \pi i}{a^{3 / 4}} I_{10}(a, 0,0) .
$$

Using the fact that $\hat{\psi}_{1}$ is odd, we have that

$$
\begin{aligned}
\lim _{a \rightarrow 0^{+}} 2 \pi i a^{-\frac{3}{4}} I_{1}(a, 0,0) \\
\quad=\sec \theta_{0} \int_{0}^{\infty} \hat{\psi}_{1}(\rho) \int_{-1}^{1} e^{\frac{\pi i \rho}{2 A} t^{2}} \hat{\psi}_{2}(t) d t \int_{-\infty}^{\infty} e^{-2 \pi i \rho A \cos \theta_{0} u^{2}} d u d \rho \\
\quad+\sec \theta_{0} \int_{0}^{\infty} \hat{\psi}_{1}(\rho) \int_{-1}^{1} e^{-\frac{\pi i \rho}{2 A} t^{2}} \hat{\psi}_{2}(t) d t \int_{-\infty}^{\infty} e^{2 \pi i \rho A \cos \theta_{0} u^{2}} d u d \rho \\
\quad=\sec \theta_{0} \int_{0}^{\infty} \hat{\psi}_{1}(\rho) \int_{-1}^{1} \hat{\psi}_{2}(t) 2 \Re\left\{e^{\frac{\pi i \rho}{2 A} t^{2}} \int_{-\infty}^{\infty} e^{-2 \pi i \rho A \cos \theta_{0} u^{2}} d u\right\} d t d \rho .
\end{aligned}
$$

Recalling the formulas of Fresnel integrals

$$
\int_{-\infty}^{\infty} \cos \left(\frac{\pi}{2} x^{2}\right) d x=\int_{-\infty}^{\infty} \sin \left(\frac{\pi}{2} x^{2}\right) d x=1
$$

it follows that

$$
\int_{-\infty}^{\infty} \cos \left(2 \pi \rho A \cos \theta_{0} x^{2}\right) d x=\int_{-\infty}^{\infty} \sin \left(2 \pi \rho A \cos \theta_{0} x^{2}\right) d x=\frac{1}{2 \sqrt{\rho A \cos \theta_{0}}}
$$

Thus, we conclude that

$$
\begin{aligned}
& \lim _{a \rightarrow 0^{+}} 2 \pi i a^{-\frac{3}{4}} I_{1}(a, 0,0) \\
& =\frac{\left(\sec \theta_{0}\right)^{\frac{3}{2}}}{\sqrt{A}} \int_{0}^{\infty} \frac{\hat{\psi}_{1}(\rho)}{\sqrt{\rho}}\left(\int_{-1}^{1} \cos \left(\frac{\pi \rho}{2 A} t^{2}\right) \hat{\psi}_{2}(t) d t+\int_{-1}^{1} \sin \left(\frac{\pi \rho}{2 A} t^{2}\right) \hat{\psi}_{2}(t) d t\right) d \rho .
\end{aligned}
$$

The last expression is strictly positive by Lemma 1 and by the properties of $\hat{\psi}_{1}$.

Case 2: $A=0$. Since, in this case, $G_{0}(u)=-u \tan \theta_{0}$ and, hence, $G_{0}^{\prime}(u)=$ $-\tan \theta_{0}$, it follows that

$$
\begin{aligned}
& \int_{-\varepsilon}^{\varepsilon} e^{-2 \pi i \frac{\rho}{a}\left(G_{0}(u) \cos \theta+u \sin \theta\right)}\left(-\cos \theta+G_{0}^{\prime}(u) \sin \theta\right) d u \\
& =-\left(\cos \theta+\sin \theta \tan \theta_{0}\right) \int_{-\varepsilon}^{\varepsilon} e^{-2 \pi i \frac{\rho}{a} u \sin \theta} d u .
\end{aligned}
$$

Using this observation, a direct calculation yields that 


$$
\begin{aligned}
& 2 \pi i I_{1}(a, 0,0) \\
= & -a^{-\frac{1}{4}} \int_{0}^{\infty} \int_{0}^{2 \pi} \hat{\psi}_{1}(\rho \cos \theta) \hat{\psi}_{2}\left(a^{-\frac{1}{2}}\left(\tan \theta-\tan \theta_{0}\right)\right) \\
& \times\left(-\left(\cos \theta+\sin \theta \tan \theta_{0}\right) \int_{-\varepsilon}^{\varepsilon} e^{-2 \pi i \frac{\rho}{a}\left(\sin \theta-\cos \theta \tan \theta_{0}\right) u} d u\right) d \theta d \rho \\
= & a^{-\frac{1}{4}} \int_{0}^{\infty} \int_{-\varepsilon}^{\varepsilon} \int_{0}^{2 \pi} \hat{\psi}_{1}(\rho \cos \theta) \hat{\psi}_{2}\left(a^{-\frac{1}{2}}\left(\tan \theta-\tan \theta_{0}\right)\right) \\
& \times e^{-2 \pi i \frac{\rho}{a} \sin \theta u}\left(\cos \theta+\sin \theta \tan \theta_{0}\right) d \theta d u d \rho \\
= & a^{-\frac{1}{4}} \int_{0}^{\infty} \int_{-\varepsilon}^{\varepsilon} \int_{-\frac{\pi}{2}}^{\frac{\pi}{2}} \hat{\psi}_{1}(\rho \cos \theta) \hat{\psi}_{2}\left(a^{-\frac{1}{2}}\left(\left(\tan \theta-\tan \theta_{0}\right)\right)\right. \\
& \times e^{-2 \pi i \frac{\rho}{a}\left(\sin \theta-\cos \theta \tan \theta_{0}\right) u}\left(\cos \theta+\sin \theta \tan \theta_{0}\right) d \theta d u d \rho \\
& +a^{-\frac{1}{4}} \int_{0}^{\infty} \int_{-\varepsilon}^{\varepsilon} \int_{-\frac{\pi}{2}}^{\frac{\pi}{2}} \hat{\psi}_{1}(\rho \cos \theta) \hat{\psi}_{2}\left(a^{-\frac{1}{2}}\left(\tan \theta-\tan \theta_{0}\right)\right) \\
& \times e^{2 \pi i \frac{\rho}{a}\left(\sin \theta-\cos \theta \tan \theta_{0}\right) u}\left(\cos \theta+\sin \theta \tan \theta_{0}\right) d \theta d u d \rho
\end{aligned}
$$

Using the change of variables $t=a^{-\frac{1}{2}}\left(\tan \theta-\tan \theta_{0}\right)$ and $a^{-\frac{1}{2}} u=u^{\prime}$, from the last set of equalities we obtain that

$$
\begin{aligned}
& \lim _{a \rightarrow 0^{+}} 2 \pi i a^{-\frac{3}{4}} I_{1}(a, 0,0) \\
= & \int_{0}^{\infty} \int_{0}^{\infty} \hat{\psi}_{1}(\rho) \int_{-1}^{1} \hat{\psi}_{2}(t) e^{-2 \pi i \cos \theta_{0} \rho t u} d t d u d \rho \\
& +\int_{0}^{\infty} \int_{0}^{\infty} \hat{\psi}_{1}(\rho) \int_{-1}^{1} \hat{\psi}_{2}(t) e^{2 \pi i \cos \theta_{0} \rho t u} d t d u d \rho \\
= & \sec \theta_{0} \hat{\psi}_{2}(0) \int_{0}^{\infty} \frac{\hat{\psi}_{1}(\rho)}{\rho} d \rho>0 .
\end{aligned}
$$

This completes the proof of (iii) and, together with it, the proof of Theorem 2.

We now prove Theorem 3. Notice that the proofs of parts (i) and (iii) are obtained by modifying the arguments used in (ii) and (iii) of the proof above.

Proof of Theorem 3.

- Part (i). The proof follows essentially the same ideas as in the proof of (ii) of Theorem 2.

Assume that $s=s_{0}$ does not correspond to any of the normal directions of $\partial S$ at $p=(0,0)$. As in the the proof of Theorem 2, we write $s_{0}=\tan \theta_{0}$, where we assume that $\left|\theta_{0}\right| \leq \frac{\pi}{4}$.

Breaking the interval $\left[-\frac{\pi}{2}, \frac{3 \pi}{2}\right]$ into $\left[-\frac{\pi}{2}, \frac{\pi}{2}\right]$ and $\left[\frac{\pi}{2}, \frac{3 \pi}{2}\right]$, and changing the variable $\theta=\theta^{\prime}+\pi$ for the integral on $\left[\frac{\pi}{2}, \frac{3 \pi}{2}\right]$, we can write $I_{1}$, given by (24), as

$$
I_{1}\left(a, s_{0},(0,0)\right)=I_{11}\left(a, s_{0},(0,0)\right)+I_{12}\left(a, s_{0},(0,0)\right),
$$


where, for $j=1,2$,

$$
\begin{aligned}
& I_{1 j}\left(a, s_{0},(0,0)\right) \\
& \quad=-\frac{a^{-\frac{1}{4}}}{2 \pi i} \int_{0}^{\infty} \int_{-\frac{\pi}{2}}^{\frac{\pi}{2}} \hat{\psi}_{1}(\rho \cos \theta) \hat{\psi}_{2}\left(a^{-1 / 2}\left(\tan \theta-\tan \theta_{0}\right)\right) K_{j}(a, \rho, \theta) d \theta d \rho,
\end{aligned}
$$

and

$$
K_{j}(a, \rho, \theta)=K_{j 1}(a, \rho, \theta)+K_{j 2}(a, \rho, \theta),
$$

with

$$
\begin{aligned}
& K_{11}(a, \rho, \theta)=\int_{1-\varepsilon}^{1} e^{-2 \pi i \frac{\rho}{a} \Theta(\theta) \cdot \alpha(t)} \Theta(\theta) \cdot \mathbf{n}(t) d t \\
& K_{12}(a, \rho, \theta)=\int_{1}^{1+\varepsilon} e^{-2 \pi i \frac{\rho}{a} \Theta(\theta) \cdot \alpha(t)} \Theta(\theta) \cdot \mathbf{n}(t) d t \\
& K_{21}(a, \rho, \theta)=\int_{1-\varepsilon}^{1} e^{2 \pi i \frac{\rho}{a} \Theta(\theta) \cdot \alpha(t)} \Theta(\theta) \cdot \mathbf{n}(t) d t \\
& K_{22}(a, \rho, \theta)=\int_{1}^{1+\varepsilon} e^{2 \pi i \frac{\rho}{a} \Theta(\theta) \cdot \alpha(t)} \Theta(\theta) \cdot \mathbf{n}(t) d t
\end{aligned}
$$

By the support condition on $\hat{\psi}_{2}$, we have that $\theta \rightarrow \theta_{0}$ as $a \rightarrow 0$. Since $s_{0}=\tan \theta_{0}$ does not correspond to any of the normal directions of $\partial S$ at $(0,0)$, it follows that $\Theta\left(\theta_{0}\right) \cdot \alpha^{\prime}(1) \neq 0$. Hence, for $a$ sufficiently small (in which case $\theta$ is near $\theta_{0}$ ), there is an $\varepsilon>0$ sufficiently small, such that $\Theta(\theta) \cdot \alpha^{\prime}(t) \neq 0$ for all $\theta$ near $\theta_{0}$ and $t \in[1-\varepsilon, 1+\varepsilon]$. Next, writing

$$
e^{-2 \pi i \frac{\rho}{a} \Theta(\theta) \cdot \alpha(t)}=\frac{-a}{2 \pi i \rho \Theta(\theta) \cdot \alpha^{\prime}(t)}\left(e^{-2 \pi i \frac{\rho}{a} \Theta(\theta) \cdot \alpha(t)}\right)^{\prime},
$$

and then integrating by parts twice the integral $K_{11}$ with respect to $t$, we obtain

$$
\begin{aligned}
K_{11}(a, \rho, \theta) & =-\frac{a}{2 \pi i \rho} \int_{1-\varepsilon}^{1}\left(e^{-2 \pi i \frac{\rho}{a} \Theta(\theta) \cdot \alpha(t)}\right)^{\prime} \frac{\Theta(\theta) \cdot \mathbf{n}(t)}{\Theta(\theta) \cdot \alpha^{\prime}(t)} d t \\
& =K_{111}(a, \rho, \theta)+K_{112}(a, \rho, \theta)+K_{113}(a, \rho, \theta)+O\left(a^{3}\right)
\end{aligned}
$$

where

$$
\begin{aligned}
K_{111}(a, \rho, \theta) & =-\frac{a}{2 \pi i \rho} e^{-2 \pi i \frac{\rho}{a} \Theta(\theta) \cdot \alpha\left(1^{-}\right)} \frac{\Theta(\theta) \cdot \mathbf{n}\left(1^{-}\right)}{\Theta(\theta) \cdot \alpha^{\prime}\left(1^{-}\right)} \\
K_{112}(a, \rho, \theta) & =\frac{a}{2 \pi i \rho} e^{-2 \pi i \frac{\rho}{a} \Theta(\theta) \cdot \alpha(1-\varepsilon)} \frac{\Theta(\theta) \cdot \mathbf{n}(1-\varepsilon)}{\Theta(\theta) \cdot \alpha^{\prime}(1-\varepsilon)} \\
K_{113}(a, \rho, \theta) & =\left.\frac{a^{2}}{(2 \pi i \rho)^{2}}\left(e^{-2 \pi i \frac{\rho}{a} \Theta(\theta) \cdot \alpha(t)} \frac{1}{\Theta(\theta) \cdot \alpha^{\prime}(t)}\left(\frac{\Theta(\theta) \cdot \mathbf{n}(t)}{\Theta(\theta) \cdot \alpha^{\prime}(t)}\right)^{\prime}\right)\right|_{1-\varepsilon} ^{1} .
\end{aligned}
$$

Similarly, one can write 


$$
K_{12}(a, \rho, \theta)=K_{121}(a, \rho, \theta)+K_{122}(a, \rho, \theta)+K_{123}(a, \rho, \theta)+O\left(a^{3}\right) .
$$

Accordingly, we write

$$
I_{11}\left(a, s_{0}, p\right)=I_{111}\left(a, s_{0}, p\right)+I_{112}\left(a, s_{0}, p\right)+I_{113}\left(a, s_{0}, p\right)+O\left(a^{3}\right)
$$

where, for $l=1,2,3$,

$$
\begin{aligned}
I_{11 l}\left(a, s_{0}, p\right)= & -\frac{a^{-\frac{1}{4}}}{2 \pi i} \int_{0}^{\infty} \int_{0}^{2 \pi} \hat{\psi}_{1}(\rho \cos \theta) \hat{\psi}_{2}\left(a^{-1 / 2}\left(\tan \theta-\tan \theta_{0}\right)\right) \times \\
& \times\left(K_{11 l}(a, \rho, \theta)+K_{12 l}(a, \rho, \theta)\right) d \theta d \rho .
\end{aligned}
$$

Similarly, for the integral $I_{12}$ we write

$$
I_{12}\left(a, s_{0}, p\right)=I_{121}\left(a, s_{0}, p\right)+I_{122}\left(a, s_{0}, p\right)+I_{123}\left(a, s_{0}, p\right)+O\left(a^{3}\right),
$$

where, for $l=1,2,3$,

$$
\begin{aligned}
I_{12 l}\left(a, s_{0}, p\right)= & -\frac{a^{-\frac{1}{4}}}{2 \pi i} \int_{0}^{\infty} \int_{0}^{2 \pi} \hat{\psi}_{1}(\rho \cos \theta) \hat{\psi}_{2}\left(a^{-1 / 2}\left(\tan \theta-\tan \theta_{0}\right)\right) \times \\
& \times\left(K_{21 l}(a, \rho, \theta)+K_{22 l}(a, \rho, \theta)\right) d \theta d \rho,
\end{aligned}
$$

and the terms $K_{21 l}, K_{22 l}$ are constructed as the corresponding terms $K_{11 l}, K_{12 l}$. A direct computation shows that

$$
K_{111}(a, \rho, \theta)+K_{121}(a, \rho, \theta)+K_{211}(a, \rho, \theta)+K_{221}(a, \rho, \theta)=0,
$$

and this implies that ${ }^{1}$

$$
I_{111}\left(a, s_{0}, p\right)+I_{121}\left(a, s_{0}, p\right)=0 .
$$

Since $\partial S$ is simple, it follows that $\alpha(1-\varepsilon) \neq(0,0)$ and $\alpha(1+\varepsilon) \neq(0,0)$. Therefore, by the argument used in the proof of Lemma 2, it follows that, for any $N>0$,

$$
\left|I_{112}\left(a, s_{0}, p\right)\right| \leq C_{N} a^{N}, \quad \text { as } a \rightarrow 0 .
$$

Similarly, one has

$$
\left|I_{122}\left(a, s_{0}, p\right)\right| \leq C_{N} a^{N}, \quad \text { as } a \rightarrow 0 .
$$

It only remains to analyze the terms $I_{113}, I_{123}$. To do that, notice that each one of the elements $K_{113}, K_{123}, K_{213}, K_{223}$, is made out of two terms, one at $t=1 \pm \varepsilon$ and one at $t=1$. As for the integrals $I_{112}\left(a, s_{0}, p\right)$ and $I_{122}\left(a, s_{0}, p\right)$, also in this case the $K$ terms evaluated at $t=1 \pm \varepsilon$ have fast asymptotic decay as $a \rightarrow 0$, and can be included in negligible part $O\left(a^{3}\right)$. Thus, in order to determine the asymptotic decay rate for $I_{113}\left(a, s_{0}, p\right)+I_{123}\left(a, s_{0}, p\right)$, one only needs to analyze the corresponding $K$

\footnotetext{
${ }^{1}$ Notice: the assumption that $\hat{\psi}_{1}$ is odd makes this cancellation possible. By contrast, the generating function of the curvelet system, which is defined in polar coordinates, does not share this property.
} 
terms corresponding to $t=1$. To do that, let $\kappa(t)$ be the curvature of $\partial S$ at $\alpha(t)$. By the Frenet's formula [3], we have that

$$
\alpha^{\prime \prime}(t)=\kappa(t) \mathbf{n}(t), \quad \mathbf{n}^{\prime}(t)=-\kappa(t) \alpha^{\prime}(t) .
$$

Hence, using these equalities and the fact the the pair $\left\{\alpha^{\prime}(t), \mathbf{n}(t)\right\}$ is an orthonormal basis in $\mathbb{R}^{2}$, we have:

$$
\begin{aligned}
& \left(\frac{\Theta(\theta) \cdot \mathbf{n}(t)}{\Theta(\theta) \cdot \alpha^{\prime}(t)}\right)^{\prime} \\
& =\frac{\left(\Theta(\theta) \cdot \mathbf{n}^{\prime}(t)\right)\left(\Theta(\theta) \cdot \alpha^{\prime}(t)\right)-\left(\Theta(\theta) \cdot \alpha^{\prime \prime}(t)\right)(\Theta(\theta) \cdot \mathbf{n}(t))}{\left(\Theta(\theta) \cdot \alpha^{\prime}(t)\right)^{2}} \\
& =-\frac{\kappa(t)\left(\left(\Theta(\theta) \cdot \alpha^{\prime}(t)\right)\left(\Theta(\theta) \cdot \alpha^{\prime}(t)\right)+(\Theta(\theta) \cdot \mathbf{n}(t))(\Theta(\theta) \cdot \mathbf{n}(t))\right)}{\left(\Theta(\theta) \cdot \alpha^{\prime}(t)\right)^{2}} \\
& =-\frac{\kappa(t)|\Theta(\theta)|^{2}}{\left(\Theta(\theta) \cdot \alpha^{\prime}(t)\right)^{2}}=-\frac{\kappa(t)}{\left(\Theta(\theta) \cdot \alpha^{\prime}(t)\right)^{2}} .
\end{aligned}
$$

It follows from the above observations that

$$
\begin{aligned}
& \lim _{a \rightarrow 0^{+}}\left(\frac{\rho}{a}\right)^{2}\left(K_{113}^{-}\left(a, s_{0}, p\right)+K_{123}^{+}\left(a, s_{0}, p\right)\right) \\
& =\frac{1}{(2 \pi i)^{2}}\left(\frac{\kappa\left(1^{+}\right)}{\left(\Theta\left(\theta_{0}\right) \cdot \alpha^{\prime}\left(1^{+}\right)\right)^{3}}-\frac{\kappa\left(1^{-}\right)}{\left(\Theta\left(\theta_{0}\right) \cdot \alpha^{\prime}\left(1^{-}\right)\right)^{3}}\right) .
\end{aligned}
$$

Similarly, one has

$$
\begin{aligned}
& \lim _{a \rightarrow 0^{+}}\left(\frac{\rho}{a}\right)^{2}\left(K_{213}^{-}\left(a, s_{0}, p\right)+K_{223}^{+}\left(a, s_{0}, p\right)\right) \\
& =\frac{1}{(2 \pi i)^{2}}\left(\frac{\kappa\left(1^{+}\right)}{\left(\Theta\left(\theta_{0}\right) \cdot \alpha^{\prime}\left(1^{+}\right)\right)^{3}}-\frac{\kappa\left(1^{-}\right)}{\left(\Theta\left(\theta_{0}\right) \cdot \alpha^{\prime}\left(1^{-}\right)\right)^{3}}\right) .
\end{aligned}
$$

Finally, by making the change of variables $u=a^{-\frac{1}{2}}\left(\tan \theta-\tan \theta_{0}\right)$ in $I_{113}$ and $I_{123}$, and applying (27) and (28), we obtain

$$
\lim _{a \rightarrow 0^{+}} a^{-\frac{9}{4}}\left(I_{113}\left(a, s_{0}, p\right)+I_{123}\left(a, s_{0}, p\right)\right)=-A,
$$

where

$$
\begin{gathered}
A=\frac{\cos ^{2} \theta_{0}}{2 \pi^{2}}\left(\frac{\kappa\left(1^{+}\right)}{\left(\Theta\left(\theta_{0}\right) \cdot \alpha^{\prime}\left(1^{+}\right)\right)^{3}}-\frac{\kappa\left(1^{-}\right)}{\left(\Theta\left(\theta_{0}\right) \cdot \alpha^{\prime}\left(1^{-}\right)\right)^{3}}\right) \\
\quad \times \int_{0}^{\infty} \hat{\psi}_{1}\left(\rho \cos \theta_{0}\right) d \rho \int_{-1}^{1} \hat{\psi}_{2}(u) d u<\infty .
\end{gathered}
$$

This completes the proof of part (i). 
- Part (ii). It is sufficient to show that, if $p$ is a corner point of the second type, $A \neq 0$, where $A$ is given by (29).

In fact, we have that

$$
\alpha^{\prime}\left(1^{+}\right)=\alpha^{\prime}\left(1^{-}\right) \quad \text { or } \quad \alpha^{\prime}\left(1^{+}\right)=-\alpha^{\prime}\left(1^{-}\right) .
$$

If $\alpha^{\prime}\left(1^{+}\right)=\alpha^{\prime}\left(1^{-}\right)$, from $\kappa\left(1^{+}\right) \neq \kappa\left(1^{-}\right)$, we have

$$
A=\frac{\cos ^{2} \theta_{0}}{2 \pi^{2}} \frac{\kappa\left(1^{+}\right)-\kappa\left(1^{-}\right)}{\left(\Theta\left(\theta_{0}\right) \cdot \alpha^{\prime}(1)\right)^{3}} \int_{0}^{\infty} \hat{\psi}_{1}\left(\rho \cos \theta_{0}\right) d \rho \int_{-1}^{1} \hat{\psi}_{2}(u) d u \neq 0 .
$$

If on the other hand, $\alpha^{\prime}\left(1^{+}\right)=-\alpha^{\prime}\left(1^{-}\right)$, then it follows that $\mathbf{n}\left(1^{+}\right)=-\mathbf{n}\left(1^{-}\right)$. Since $\alpha^{\prime \prime}\left(1^{+}\right)=-\kappa\left(1^{+}\right) \mathbf{n}\left(1^{-}\right)$and $\alpha^{\prime \prime}\left(1^{-}\right)=\kappa\left(1^{-}\right) \mathbf{n}\left(1^{-}\right)$, and since $\kappa\left(1^{+}\right) \neq \kappa\left(1^{-}\right)$, we see that it is not possible to have $\kappa\left(1^{+}\right)=\kappa\left(1^{-}\right)=0$. Since we know $\kappa\left(1^{+}\right) \geq 0$, and $\kappa\left(1^{-}\right) \geq 0$, it follows that $\kappa\left(1^{+}\right)+\kappa\left(1^{-}\right)>0$. Thus, also in this case we have

$$
A=\frac{\cos ^{2} \theta_{0}}{2 \pi^{2}} \frac{\kappa\left(1^{+}\right)+\kappa\left(1^{-}\right)}{\left(\Theta\left(\theta_{0}\right) \cdot \alpha^{\prime}\left(1^{+}\right)\right)^{3}} \int_{0}^{\infty} \hat{\psi}_{1}\left(\rho \cos \theta_{0}\right) d \rho \int_{-1}^{1} \hat{\psi}_{2}(u) d u \neq 0 .
$$

This completes the proof of part (ii).

- Part (iii). By the assumptions on the corner points, it follows that if $s$ corresponds to $\mathscr{C}^{-}$at $p$, then it cannot correspond to $\mathscr{C}^{+}$at $p$. From part (i), we see that it is enough to consider $\mathscr{C}^{-}$or $\mathscr{C}^{+}$. Thus, we may assume that $s$ corresponds to the outer normal direction of $\mathscr{C}^{+}$at $p=(0,0)$. Since the argument for this case is very similar to the one for (iii) of Theorems 2 , to save the notations here we assume $\theta_{0}=0$ so that $s=0$.

Let $S, G, S_{0}, G_{0}$ be defined as in Lemma 3. Thus, according to Lemma 3 , on $\mathscr{C}^{+}$, we one can use $G_{0}(u)$ to replace $G^{+}(u)$ since the approximation error is $o\left(a^{\frac{3}{4}}\right)$. For simplicity, in the following we will use $G$ to denote $G^{+}$.

Using polar coordinates we can express $I_{1}(a, 0,0)$, evaluated on $S_{0}$, as

$$
\begin{aligned}
& I_{1}(a, 0,0)=-\frac{1}{2 \pi i a^{\frac{1}{4}}} \int_{0}^{\infty} \int_{0}^{2 \pi} \hat{\psi}_{1}(\rho \cos \theta) \hat{\psi}_{2}\left(a^{-\frac{1}{2}} \tan \theta\right) \int_{0}^{\varepsilon} e^{-2 \pi i \frac{\rho}{a}\left(\cos \theta G_{0}(u)+\sin \theta u\right)} \\
& \times\left(-\cos \theta+\sin \theta G_{0}^{\prime}(u)\right) d u d \theta d \rho .
\end{aligned}
$$

By Lemma 2 and Lemma 3, to complete the proof it is sufficient to show

$$
\lim _{a \rightarrow 0^{+}} a^{-\frac{3}{4}} I_{1}(a, 0,0) \neq 0 .
$$

Since $\theta_{0}=0$, we have $\mathbf{n}(p)=(1,0)$. It follows that $G_{0}(0)=0, G_{0}^{\prime}(0)=0$ so that $G_{0}(u)=\frac{1}{2} G^{\prime \prime}(0) u^{2}$. Let $A=\frac{1}{2} G^{\prime \prime}(0)$. We will consider separately the case $A \neq 0$ and $A=0$.

Case 1: $A \neq 0$. In this case, we may assume that $A>0$, since the case $A<0$ is similar. We have that 


$$
\begin{aligned}
& \int_{0}^{\varepsilon} e^{-2 \pi i \frac{\rho}{a}\left(G_{0}(u) \cos \theta+u \sin \theta\right)}\left(-\cos \theta+G_{0}^{\prime}(u) \sin \theta\right) d u \\
& =e^{\frac{\rho \pi i}{a} \frac{\sin ^{2} \theta}{2 \cos \theta A}} \int_{0}^{\varepsilon} e^{-2 \pi i \frac{\rho}{a} \cos \theta A\left(u-u_{\theta}\right)^{2}}(-\cos \theta+2 A u \sin \theta) d u \\
& =K_{0}(\theta, a)+K_{1}(\theta, a),
\end{aligned}
$$

where $u_{\theta}=-\frac{\sin \theta}{2 A \cos \theta}$ and

$$
\begin{aligned}
& K_{0}(\theta, a)=-\cos \theta e^{\frac{\rho \pi i}{a} \frac{\sin ^{2} \theta}{2 A \cos \theta}} \int_{0}^{\varepsilon} e^{-2 \pi i \frac{\rho}{a} \cos \theta A\left(u-u_{\theta}\right)^{2}} d u \\
& K_{1}(\theta, a)=2 A \sin \theta e^{\frac{\rho \pi i}{a} \frac{\sin ^{2} \theta}{2 A \cos \theta}} \int_{0}^{\varepsilon} e^{-2 \pi i \frac{\rho}{a} \cos \theta A\left(u-u_{\theta}\right)^{2}} u d u .
\end{aligned}
$$

In the expression of $I_{1}$, the interval $[0,2 \pi]$ of the integral in $\theta$ can be broken into the subintervals $\left[-\frac{\pi}{2}, \frac{\pi}{2}\right]$ and $\left[\frac{\pi}{2}, \frac{3 \pi}{2}\right]$. On $\left[\frac{\pi}{2}, \frac{3 \pi}{2}\right]$, we let $\theta^{\prime}=\theta-\pi$ so that $\theta^{\prime} \in\left[-\frac{\pi}{2}, \frac{\pi}{2}\right]$ and that $\sin \theta=-\sin \theta^{\prime}, \cos \theta=-\cos \theta^{\prime}$. Using this observation and the fact that $\hat{\psi}_{1}$ is an odd function, it follows that $I_{1}(a, 0,0)=I_{10}(a, 0,0)+I_{11}(a, 0,0)$, where for $j=0,1$,

$$
\begin{aligned}
& I_{1 j}(a, 0,0)=-\frac{1}{2 \pi i a^{\frac{1}{4}}} \int_{0}^{\infty} \int_{-\frac{\pi}{2}}^{\frac{\pi}{2}} \hat{\psi}_{1}(\rho \cos \theta) \hat{\psi}_{2}\left(a^{-\frac{1}{2}}(\tan \theta)\right) K_{j}(\theta, a) d \theta d \rho . \\
& +\frac{1}{2 \pi i a^{\frac{1}{4}}} \int_{0}^{\infty} \int_{-\frac{\pi}{2}}^{\frac{\pi}{2}} \hat{\psi}_{1}(\rho \cos \theta) \hat{\psi}_{2}\left(a^{-\frac{1}{2}}(\tan \theta)\right) K_{j}(\theta+\pi, a) d \theta d \rho .
\end{aligned}
$$

For $\theta \in\left(-\frac{\pi}{2}, \frac{\pi}{2}\right)$, let $t=a^{-\frac{1}{2}} \tan \theta$ (and $a^{-\frac{1}{2}} u=u^{\prime}$ ). Recall that as $a \rightarrow 0, \theta$ approaches $\theta_{0}=0$ so that $\cos \theta \rightarrow 1$. We have

$$
\begin{aligned}
\lim _{a \rightarrow 0^{+}} a^{-\frac{1}{2}} K_{0}(\theta, a) & =-e^{\frac{i \pi \rho}{2 A} t^{2}} \int_{0}^{\infty} e^{-2 \pi i \rho A\left(u-\frac{t}{2 A}\right)^{2}} d u \\
& =-e^{\frac{i \pi \rho}{2 A} t^{2}} \int_{0}^{\infty} e^{-2 \pi i \rho A u^{2}} d u-e^{\frac{i \pi \rho}{2 A} t^{2}} \int_{-\frac{t}{2 A}}^{0} e^{-2 \pi i \rho A u^{2}} d u .
\end{aligned}
$$

Similarly, we have

$$
\lim _{a \rightarrow 0^{+}} a^{-\frac{1}{2}} K_{0}(\theta+\pi, a)=-e^{-\frac{i \pi \rho}{2 A} t^{2}} \int_{0}^{\infty} e^{2 \pi i \rho A u^{2}} d u+e^{-\frac{i \pi \rho}{2 A} t^{2}} \int_{-\frac{t}{2 A}}^{0} e^{2 \pi i \rho A u^{2}} d u .
$$

Since $\sin \theta=O\left(a^{\frac{1}{2}}\right)$ (due to the conditions on the support of $\left.\hat{\psi}\right)$, based on the calculation of $K_{0}(\theta, a)$ and $K_{0}(\theta+\pi, a)$, it follows that $K_{1}(\theta, a)=O(a), K_{1}(\theta+\pi, a)=$ $O(a)$. Thus,

$$
\lim _{a \rightarrow 0^{+}} \frac{2 \pi i}{a^{3 / 4}} I_{1}(a, 0,0)=\lim _{a \rightarrow 0^{+}} \frac{2 \pi i}{a^{3 / 4}} I_{10}(a, 0,0) .
$$

Finally, using the facts that $\hat{\psi}_{2}$ is even, that the function $\int_{-\frac{t}{2 A}}^{0} e^{2 \pi i \rho A u^{2}} d u$ is an odd function of $t$, and the formulas of Fresnel integral as in the proof of Theorem 2, we 
conclude that

$$
\begin{aligned}
& \lim _{a \rightarrow 0^{+}} 2 \pi i a^{-\frac{3}{4}} I_{1}(a, 0,0)=\int_{0}^{\infty} \hat{\psi}_{1}(\rho) \int_{-1}^{1} e^{\frac{\pi i \rho}{2 A} t^{2}} \hat{\psi}_{2}(t) d t \int_{0}^{\infty} e^{-2 \pi i \rho A u^{2}} d u d \rho \\
& +\int_{0}^{\infty} \hat{\psi}_{1}(\rho) \int_{-1}^{1} e^{-\frac{\pi i \rho}{2 A} t^{2}} \hat{\psi}_{2}(t) d t \int_{0}^{\infty} e^{2 \pi i \rho A u^{2}} d u d \rho \\
& =\frac{1}{\sqrt{A}} \int_{0}^{\infty} \frac{\hat{\psi}_{1}(\rho)}{\sqrt{\rho}}\left(\int_{-1}^{1} \cos \left(\frac{\pi i \rho}{2 A} t^{2}\right) \hat{\psi}_{2}(t) d t+\int_{-1}^{1} \sin \left(\frac{\pi i \rho}{2 A} t^{2}\right) \hat{\psi}_{2}(t) d t\right) d \rho
\end{aligned}
$$

The last expression is strictly positive by Lemma 1 , and by the properties of $\hat{\psi}_{1}$.

Case 2: $A=0$. Since, in this case, $G_{0}(u)=0$, it follows that

$$
\int_{0}^{\varepsilon} e^{-2 \pi i \frac{\rho}{a}\left(G_{0}(u) \cos \theta+u \sin \theta\right)}\left(-\cos \theta+G_{0}^{\prime}(u) \sin \theta\right) d u=-\cos \theta \int_{0}^{\varepsilon} e^{-2 \pi i \frac{\rho}{a} u \sin \theta} d u .
$$

It follows that

$$
\begin{aligned}
& 2 \pi i I_{1}(a, 0,0) \\
& =-a^{-\frac{1}{4}} \int_{0}^{\infty} \int_{0}^{2 \pi} \hat{\psi}_{1}(\rho \cos \theta) \hat{\psi}_{2}\left(a^{-\frac{1}{2}} \tan \theta\right)\left(-\cos \theta \int_{0}^{\varepsilon} e^{-2 \pi i \frac{\rho}{a} \sin \theta u} d u\right) d \theta d \rho \\
& =a^{-\frac{1}{4}} \int_{0}^{\infty} \int_{0}^{\varepsilon} \int_{0}^{2 \pi} \hat{\psi}_{1}(\rho \cos \theta) \hat{\psi}_{2}\left(a^{-\frac{1}{2}} \tan \theta\right) e^{-2 \pi i \frac{\rho}{a} \sin \theta u} \cos \theta d \theta d u d \rho \\
& =a^{-\frac{1}{4}} \int_{0}^{\infty} \int_{0}^{\varepsilon} \int_{-\frac{\pi}{2}}^{\frac{\pi}{2}} \hat{\psi}_{1}(\rho \cos \theta) \hat{\psi}_{2}\left(a^{-\frac{1}{2}}(\tan \theta)\right) e^{-2 \pi i \frac{\rho}{a} \sin \theta u} \cos \theta d \theta d u d \rho \\
& +a^{-\frac{1}{4}} \int_{0}^{\infty} \int_{0}^{\varepsilon} \int_{-\frac{\pi}{2}}^{\frac{\pi}{2}} \hat{\psi}_{1}(\rho \cos \theta) \hat{\psi}_{2}\left(a^{-\frac{1}{2}}(\tan \theta)\right) e^{2 \pi i \frac{\rho}{a} \sin \theta u} \cos \theta d \theta d u d \rho
\end{aligned}
$$

Using the change of variables $t=a^{-\frac{1}{2}} \tan \theta$ (and $a^{-\frac{1}{2}} u=u^{\prime}$ ) we obtain

$$
\begin{aligned}
& \lim _{a \rightarrow 0^{+}} 2 \pi i a^{-\frac{3}{4}} I_{1}(a, 0,0) \\
& =\int_{0}^{\infty} \int_{0}^{\infty} \hat{\psi}_{1}(\rho) \int_{-1}^{1} \hat{\psi}_{2}(t) e^{-2 \pi i \rho t u} d t d u d \rho+\int_{0}^{\infty} \int_{0}^{\infty} \hat{\psi}_{1}(\rho) \int_{-1}^{1} \hat{\psi}_{2}(t) e^{2 \pi i \rho t u} d t d u d \rho \\
& =\left(\hat{\psi}_{2}(0)\right)^{2} \int_{0}^{\infty} \frac{\hat{\psi}_{1}(\rho)}{\rho} d \rho>0 .
\end{aligned}
$$

This completes the proof of (iii) and, together with it, the proof of Theorem 3.

\subsection{Extensions and Generalizations}

The results presented above are limited to the analysis of the continuous shearlet transform of characteristic functions of sets. It is clear that, to provide a more re- 
alistic model for images containing edges, it would be useful to consider a more general class of compactly supported functions, which are not necessarily constant or piecewise constant.

Unfortunately, the analysis of this situation is significantly more complicated and cannot be derived directly using the techniques developed above. This is due to the fact that one main technical tool which was used to deduced Theorem 2 and 3 is the divergence theorem (cf. (22)), which allows us to conveniently express the Fourier transform of $B=\chi_{S}$, the characteristic function of a set $S$. If $\chi_{S}$ is replaced by $g \chi_{S}$, this produces a convolution in the Fourier domain, and this has a dramatic impact on all the arguments used above, even in the simplified situation where $g$ can be expanded using a Taylor polynomial.

Despite the fact that a general result for the characterization of singularities of piecewise smooth functions is not known at the moment, it is still possible to derive some useful observations which take advantage of the directional sensitivity of the continuous shearlet transform. Following the approach in [6], let $\Omega$ be a bounded open subset of $\mathbb{R}^{2}$ and assume a smooth partition

$$
\Omega=\bigcup_{n=1}^{L} \Omega_{n} \cup \Gamma
$$

where:

1. for each $n=1, \ldots, L, \Omega_{n}$ is a connected open domain;

2. each boundary $\partial_{\Omega} \Omega_{n}$ is generated by a $C^{3}$ curve $\gamma_{n}$ and each of the boundary curves $\gamma_{n}$ can be parametrized as $(\rho(\theta) \cos \theta, \rho(\theta) \sin \theta)$ where $\rho(\theta):[0,2 \pi) \rightarrow$ $[0,1]$ is a radius function;

3. $\Gamma=\bigcup_{n=1}^{L} \partial_{\Omega} \Omega_{n}$, where $\partial_{\Omega} X$ denotes the relative topological boundary in $\Omega$ of $X \subset \Omega$.

Hence, we define the space $E^{1,3}(\Omega)$ as the collection of functions which are compactly supported in $\Omega$ and have the form

$$
f(x)=\sum_{n=1}^{L} f_{n}(x) \chi_{\Omega_{n}}(x) \text { for } x \in \Omega \backslash \Gamma
$$

where, for each $n=1, \ldots, L, f_{n} \in C_{0}^{1}(\Omega)$ with $\sum_{|\alpha| \leq 1}\left\|D^{\alpha} f_{n}\right\|_{\infty} \leq C$ for some $C>0$, and the sets $\Omega_{n}$ are pairwise disjoint in measure. The functions $E^{1,3}(\Omega)$ are a variant of the cartoon-like images, where the set $\Gamma$ describes the boundaries of different objects. Similar image models are commonly used, for example, in the variational approach to image processing [2, Ch.3]. Notice that each term $u_{n}(x)=f_{n}(x) \chi_{\Omega_{n}}(x)$ models the relatively homogeneous interior of a single object and that the definition above does not specify the function value along the boundary set $\Gamma$.

For each $x$ in a $C^{3}$ component of $\Gamma$, we define the jump of $f$ at $x$, denoted by $[f]_{x}$, to be

$$
[f]_{x}=\lim _{\varepsilon \rightarrow 0^{+}} f\left(x+\varepsilon v_{x}\right)-f\left(x-\varepsilon v_{x}\right)
$$


where $v_{x}$ is an unit normal vector along $\Gamma$ at $x$. Also, for $x \in \mathbb{R}^{2}, L>0$, we denote the cube of center $x$ and side-length $2 L$ by $Q(x, L)$; that is, $Q(x, L)=[-L, L]^{2}+x$. For $k=\left(k_{1}, k_{2}\right) \in \mathbb{Z}^{2}$, let $M \in \mathbb{N}$ be sufficiently large so that each of the boundary curves $\gamma_{n}$ may be parametrized as either $\left(E\left(t_{2}\right), t_{2}\right)$ or $\left(t_{1}, E\left(t_{1}\right)\right)$ in $Q\left(\frac{k}{M}, \frac{1}{M}\right)$ if $Q\left(\frac{k}{M}, \frac{1}{M}\right) \cap$ $\Gamma \neq \emptyset$. We have the following result from [6].

Theorem 4. Let $f \in E^{1,3}(\Omega)$ and suppose that the boundary curve $\gamma_{n}$, for some $n$, is parametrized as $\left(E\left(t_{2}\right), t_{2}\right)$ in $Q\left(\frac{k}{M}, \frac{1}{M}\right)$ for some $k \in \mathbb{Z}^{2}$, and that $t=\left(E\left(t_{2}\right), t_{2}\right) \in$ $Q\left(\frac{k}{M}, \frac{1}{2 M}\right)$ for some $t_{2}$. If $s=-E^{\prime}\left(t_{2}\right)$, there exist positive constants $C_{1}$ and $C_{2}$ such that

$$
C_{1}\left|[f]_{t}\right| \leq \lim _{a \rightarrow 0^{+}} a^{-\frac{3}{4}}\left|\mathscr{S}_{\mathscr{H}} \psi(a, s, t)\right| \leq C_{2}\left|[f]_{t}\right| .
$$

If $s \neq-E^{\prime}\left(t_{2}\right)$, then

$$
\lim _{a \rightarrow 0^{+}} a^{-\frac{3}{4}}\left|\mathscr{S}_{\mathscr{H}} f(a, s, t)\right|=0 .
$$

This shows that, on the discontinuity curve, if $s$ corresponds to the normal orientation, then the continuous shearlet transform of $f$ decay as $O\left(a^{-\frac{3}{4}}\right)$, provided $\left|[f]_{t}\right| \neq 0$. However, if $s$ does not correspond to the normal orientation, we can only claim that $\mathscr{S}_{\mathscr{H}} f(a, s, t)$ decays faster than $O\left(a^{-\frac{3}{4}}\right)$.

\section{Extension to Higher Dimensions}

The characterization of boundary regions using the continuous shearlet transform extends to the 3D setting $[8,9]$. However, due to the more complicated geometry, the arguments for the 2D case do not carry over directly. More precisely, the main difficulties arise in dealing with irregular points on the boundary of solid regions, for which there are still some open problems as will be described below.

\subsection{D Continuous Shearlet Transform}

The construction of shearlet systems in $3 \mathrm{D}$ follows essentially the same ideas as the $2 \mathrm{D}$ construction. Also in this case, it is convenient to use separate shearlet systems defined in different subregions of the frequency space. This leads to the definition of three pyramid-based systems, associated with the pyramidal regions:

$$
\begin{aligned}
& \mathscr{P}_{1}=\left\{\left(\xi_{1}, \xi_{2}, \xi_{3}\right) \in \mathbb{R}^{3}:\left|\xi_{1}\right| \geq 2,\left|\frac{\xi_{2}}{\xi_{1}}\right| \leq 1 \text { and }\left|\frac{\xi_{3}}{\xi_{1}}\right| \leq 1\right\}, \\
& \mathscr{P}_{2}=\left\{\left(\xi_{1}, \xi_{2}, \xi_{3}\right) \in \mathbb{R}^{3}:\left|\xi_{1}\right| \geq 2,\left|\frac{\xi_{2}}{\xi_{1}}\right|>1 \text { and }\left|\frac{\xi_{3}}{\xi_{1}}\right| \leq 1\right\}, \\
& \mathscr{P}_{3}=\left\{\left(\xi_{1}, \xi_{2}, \xi_{3}\right) \in \mathbb{R}^{3}:\left|\xi_{1}\right| \geq 2,\left|\frac{\xi_{2}}{\xi_{1}}\right| \leq 1 \text { and }\left|\frac{\xi_{3}}{\xi_{1}}\right|>1\right\} .
\end{aligned}
$$

For $\xi=\left(\xi_{1}, \xi_{2}, \xi_{3}\right) \in \mathbb{R}^{3}, \xi_{1} \neq 0$, let $\psi^{(d)}, d=1,2,3$ be defined by 


$$
\begin{aligned}
\hat{\psi}^{(1)}(\xi) & =\hat{\psi}^{(1)}\left(\xi_{1}, \xi_{2}, \xi_{3}\right)=\hat{\psi}_{1}\left(\xi_{1}\right) \hat{\psi}_{2}\left(\frac{\xi_{2}}{\xi_{1}}\right), \hat{\psi}_{2}\left(\frac{\xi_{3}}{\xi_{1}}\right), \\
\hat{\psi}^{(2)}(\xi) & =\hat{\psi}^{(2)}\left(\xi_{1}, \xi_{2}, \xi_{3}\right)=\hat{\psi}_{1}\left(\xi_{2}\right) \hat{\psi}_{2}\left(\frac{\xi_{1}}{\xi_{2}}\right), \hat{\psi}_{2}\left(\frac{\xi_{3}}{\xi_{2}}\right), \\
\hat{\psi}^{(3)}(\xi) & =\hat{\psi}^{(3)}\left(\xi_{1}, \xi_{2}, \xi_{3}\right)=\hat{\psi}_{1}\left(\xi_{3}\right) \hat{\psi}_{2}\left(\frac{\xi_{2}}{\xi_{3}}\right), \hat{\psi}_{2}\left(\frac{\xi_{1}}{\xi_{3}}\right),
\end{aligned}
$$

where $\psi_{1}, \psi_{2}$ satisfy the same assumptions as in the 2D case. Hence, for $d=1,2,3$, the $3 D$ pyramid-based continuous shearlet systems for $L^{2}\left(\mathscr{P}_{d}\right)^{\vee}$ are the systems

$$
\left\{\psi_{a, s_{1}, s_{2}, t}^{(d)}: 0 \leq a \leq \frac{1}{4},-\frac{3}{2} \leq s_{1} \leq \frac{3}{2},-\frac{3}{2} \leq s_{2} \leq \frac{3}{2}, t \in \mathbb{R}^{3}\right\}
$$

where $\psi_{a, s_{1}, s_{2}, t}^{(d)}(x)=\left|\operatorname{det} M_{a s_{1} s_{2}}^{(d)}\right|^{-\frac{1}{2}} \psi^{(d)}\left(\left(M_{a s_{1} s_{2}}^{(d)}\right)^{-1}(x-t)\right)$, and

$$
\begin{gathered}
M_{a s_{1} s_{2}}^{(1)}=\left(\begin{array}{ccc}
a & -a^{1 / 2} s_{1} & -a^{1 / 2} s_{2} \\
0 & a^{1 / 2} & 0 \\
0 & 0 & a^{1 / 2}
\end{array}\right), M_{a s_{1} s_{2}}^{(2)}=\left(\begin{array}{ccc}
a^{1 / 2} & 0 & 0 \\
-a^{1 / 2} s_{1} & a & -a^{1 / 2} s_{2} \\
0 & 0 & a^{1 / 2}
\end{array}\right), \\
M_{a s_{1} s_{2}}^{(3)}=\left(\begin{array}{ccc}
a^{1 / 2} & 0 & 0 \\
0 & a^{1 / 2} & 0 \\
-a^{1 / 2} s_{1} & -a^{1 / 2} s_{2} & a
\end{array}\right) .
\end{gathered}
$$

Notice that the elements of the shearlet systems $\psi_{a, s_{1}, s_{2}, t}^{(d)}$ are well localized waveforms associated with various scales, controlled by $a$, various orientations, controlled by the two shear variables $s_{1}, s_{2}$ and various locations, controlled by $t$. Similar to the $2 \mathrm{D}$ case, in each pyramidal region the shearing variables are only allowed to vary over a compact set.

For $f \in L^{2}\left(\mathbb{R}^{3}\right)$, we define the $3 D$ (fine-scale) pyramid-based continuous shearlet transform $f \rightarrow \mathscr{S} \mathscr{H}_{\psi} f\left(a, s_{1}, s_{2}, t\right)$, for $a>0, s_{1}, s_{2} \in \mathbb{R}, t \in \mathbb{R}^{3}$ by

$$
\mathscr{S}_{\mathcal{H}_{\psi}} f\left(a, s_{1}, s_{2}, t\right)= \begin{cases}\left\langle f, \psi_{a, s_{1}, s_{2}, t}^{(1)}\right\rangle & \text { if }\left|s_{1}\right|,\left|s_{2}\right| \leq 1, \\ \left\langle f, \psi_{a, \frac{1}{s_{1}},}^{(2)}, \frac{s_{2}}{s_{1}}, t\right. & \text { if }\left|s_{1}\right|>1,\left|s_{2}\right| \leq\left|s_{1}\right| \\ \left\langle f, \psi_{a, s_{1}}^{(3)}, \frac{1}{s_{2}}, t\right. & \text { if }\left|s_{2}\right|>1,\left|s_{2}\right|>\left|s_{1}\right| .\end{cases}
$$

That is, depending on the values of the shearing variables, the $3 \mathrm{D}$ continuous shearlet transform corresponds to one specific pyramid-based shearlet system. As above, we are only interested in the continuous shearlet transform at"fine scales", as $a$ approaches 0 , since this is all we need for the analysis of the singularities of $f$.

\subsection{Characterization of $3 D$ Boundaries}

The $3 \mathrm{D}$ continuous shearlet transform shares the same properties as its $2 \mathrm{D}$ counterpart in terms of the ability to characterize the geometry of the set of singularities of a function or distribution $f$. In particular, it is possible to derive a characterization for the boundary set of some rather general solid regions. 


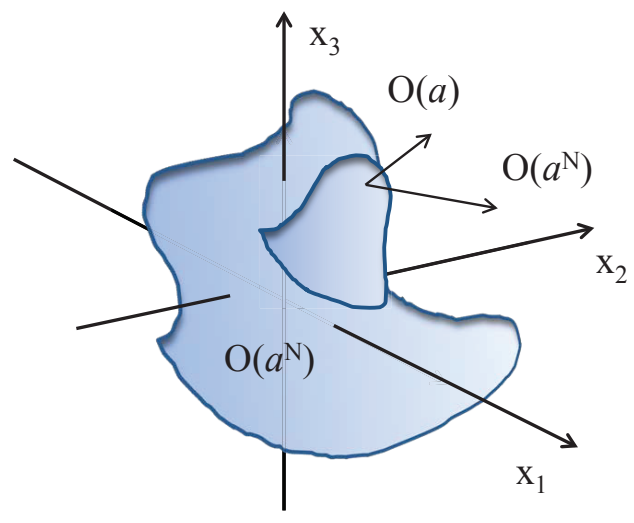

Fig. 3 General region $\Omega \subset \mathbb{R}^{3}$ with piecewise smooth boundary $\partial \Omega$. The continuous shearlet transform of $B=\chi_{\Omega}$ has rapid asymptotic decay except when the location variable $t$ is on $\partial \Omega$ and the shearing variables $\left(s_{1}, s_{2}\right)$ correspond to the normal direction at $t$; in this case $\mathscr{S} \mathscr{H}_{\psi} B\left(a, s_{1}, s_{2}, t\right) \sim a$, as $a \rightarrow 0$.

To present this results, let us define the type of surfaces which will be considered. Let $B=\chi_{\Omega}$, where $\Omega$ is a subset of $\mathbb{R}^{3}$ whose boundary $\partial \Omega$ is a 2-dimensional manifold. We say that $\partial \Omega$ is piecewise smooth if:

(i) $\partial \Omega$ is a $C^{\infty}$ manifold except possibly for finitely many separating $C^{3}$ curves on $\partial \Omega$

(ii) at each point on a separating curve, $\partial \Omega$ has exactly two outer normal vectors, which are not on the same line.

Let the outer normal vector of $\partial \Omega$ be $\mathbf{n}_{p}= \pm\left(\cos \theta_{0} \sin \phi_{0}, \sin \theta_{0} \sin \phi_{0}, \cos \phi_{0}\right)$ for some $\theta_{0} \in[0,2 \pi], \phi_{0} \in[0, \pi]$. We say that $s=\left(s_{1}, s_{2}\right)$ corresponds to the normal direction $\mathbf{n}_{p}$ if $s_{1}=a^{-\frac{1}{2}} \tan \theta_{0}, s_{2}=a^{-\frac{1}{2}} \cot \phi_{0} \sec \theta_{0}$. Notice that this definition excludes, in particular, surfaces containing cusps, such as the vertex of a cone, since at the moment no argument is known for dealing with the type of points.

The following theorem shows that the behaviour of the 3D continuous shearlet transform is consistent with the one found in dimension 2. Namely, for a bounded region in $\mathbb{R}^{3}$ whose boundary is a piecewise smooth 2-dimensional manifold, the continuous shearlet transform of $B$, denoted by $\mathscr{S}_{\mathscr{H}} \mathscr{C}_{\psi} B\left(a, s_{1}, s_{2}, t\right)$, has rapid asymptotic decay as $a \rightarrow 0$ for all locations $t \in \mathbb{R}^{3}$, except when $t$ is on the boundary of $\Omega$ 
and the orientation variables $s_{1}, s_{2}$ correspond to the normal direction of the boundary surface at $t$, or when $t$ is on a separating curve and the orientation variables $s_{1}, s_{2}$ correspond to the normal direction of the boundary surface at $t$ (see Fig. 3). Thus, as in the $2 \mathrm{D}$ case, the continuous shearlet transform provides a description of the geometry of $\partial \Omega$ through the asymptotic decay of $\mathscr{S}_{\mathscr{H}} B\left(a, s_{1}, s_{2}, t\right)$, at fine scales.

Theorem 5. Let $\Omega$ be a bounded region in $\mathbb{R}^{3}$ and denote its boundary by $\partial \Omega$. Assume that $\partial \Omega$ is a piecewise smooth 2-dimensional manifold. Let $\gamma_{j}, j=1,2, \cdots m$ be the separating curves of $\partial \Omega$. Then we have

(i) If $t \notin \partial \Omega$ then

$$
\lim _{a \rightarrow 0^{+}} a^{-N} \mathscr{S}_{\mathscr{H}_{\psi}} B\left(a, s_{1}, s_{2}, t\right)=0, \quad \text { for all } N>0 .
$$

(ii) If $t \in \partial \Omega \backslash \bigcup_{j=1}^{m} \gamma_{j}$ and $\left(s_{1}, s_{2}\right)$ does not correspond to the normal direction of $\partial \Omega$ at $t$, then

$$
\lim _{a \rightarrow 0^{+}} a^{-N} \mathscr{S}_{\mathscr{\psi}} B\left(a, s_{1}, s_{2}, t\right)=0, \quad \text { for all } N>0 .
$$

(iii) If $t \in \partial \Omega \backslash \bigcup_{j=1}^{m} \gamma_{j}$ and $s=\left(s_{1}, s_{2}\right)$ corresponds to the normal direction of $\partial \Omega$ at $t$ or $t \in \bigcup_{j=1}^{m} \gamma_{j}$ and $s=\left(s_{1}, s_{2}\right)$ corresponds to one of the two normal directions of $\partial \Omega$ at $t$, then

$$
\lim _{a \rightarrow 0^{+}} a^{-1} \mathscr{S} \mathscr{H}_{\psi} B\left(a, s_{1}, s_{2}, t\right) \neq 0 .
$$

(iv) If $t \in \gamma_{j}$ and $\left(s_{1}, s_{2}\right)$ does not correspond to the normal directions of $\partial \Omega$ at $t$, then there is a constant $C$ (possibly $C=0$ ) such that

$$
\lim _{a \rightarrow 0^{+}} a^{-3 / 2} \mathscr{S} \mathscr{H}_{\psi} B\left(a, s_{1}, s_{2}, t\right)=C .
$$

Hence, similar to the $2 \mathrm{D}$ case, the continuous shearlet transform decays rapidly away from the boundary and on the boundary, for non-normal orientations. The decay rate is only $O\left(a^{1}\right)$ at the boundary, for normal orientation. However, the situation on the separating curves of the surface is less sharp, in the sense that, for normal orientations, the decay rate is $O\left(a^{1}\right)$, but, for non-normal orientations, we can only say that the decay rate is of the order of or faster than $O\left(a^{3 / 2}\right)(C$ could be zero). However there are examples where the decay rate on a separating curve, for non-normal orientation, is exactly $O\left(a^{3 / 2}\right)(C \neq 0)$. Thus, the rate $a^{\frac{3}{2}}$ in (iv) cannot be improved. In the following, we only make a few observation about the proof. We refer the reader to [9] for a complete proof of this result.

As in the $2 \mathrm{D}$ case, the starting point is the divergence theorem, which allows us to write the Fourier transform of $B$ can be expressed as

$$
\hat{B}(\xi)=\widehat{\chi}_{\Omega}(\xi)=-\frac{1}{2 \pi i|\xi|^{2}} \int_{\partial \Omega} e^{-2 \pi i\langle\xi, x\rangle} \xi \cdot \mathbf{n}(x) d \sigma(x),
$$

where $\mathbf{n}$ is the outer normal vector to $\partial \Omega$ at $x$. Next, using spherical coordinates, we have that 


$$
\mathscr{S} \mathscr{H}_{\psi} B\left(a, s_{1}, s_{2}, t\right)=\left\langle B, \psi_{a, s_{1}, s_{2}, t}\right\rangle=I_{1}\left(a, s_{1}, s_{2}, t\right)+I_{2}\left(a, s_{1}, s_{2}, t\right),
$$

where

$$
\begin{aligned}
& I_{1}\left(a, s_{1}, s_{2}, t\right)=\int_{0}^{2 \pi} \int_{0}^{\pi} \int_{0}^{\infty} T_{1}(\rho, \theta, \phi) \overline{\hat{\psi}_{a, s_{1}, s_{2}, t}}(\rho, \theta, \phi) \rho^{2} \sin \phi d \rho d \phi d \theta \\
& I_{2}\left(a, s_{1}, s_{2}, t\right)=\int_{0}^{2 \pi} \int_{0}^{\pi} \int_{0}^{\infty} T_{2}(\rho, \theta, \phi) \overline{\hat{\psi}_{a, s_{1}, s_{2}, t}}(\rho, \theta, \phi) \rho^{2} \sin \phi d \rho d \phi d \theta
\end{aligned}
$$

and

$$
\begin{aligned}
& T_{1}(\rho, \theta, \phi)=-\frac{1}{2 \pi i \rho} \int_{P_{\varepsilon}(t)} e^{-2 \pi i \rho \Theta(\theta, \phi) \cdot x} \Theta(\theta, \phi) \cdot \mathbf{n}(x) d \sigma(x) \\
& T_{2}(\rho, \theta, \phi)=-\frac{1}{2 \pi i \rho} \int_{\partial \Omega \backslash P_{\varepsilon}(t)} e^{-2 \pi i \rho \Theta(\theta, \phi) \cdot x} \Theta(\theta, \phi) \cdot \mathbf{n}(x) d \sigma(x),
\end{aligned}
$$

where $P_{\varepsilon}(t)=\partial \Omega \cap \beta_{\varepsilon}(t)$, and $\beta_{\varepsilon}(t)$ is a ball of radius $\varepsilon$ and center $t$. Notice that $I_{2}$ is associated with the term $T_{2}$ which is evaluated away from the location $t$ of the continuous shearlet transform. Hence, a localization result similar to Lemma 2 shows that $I_{2}$ is rapidly decreasing as $a \rightarrow 0$. The rest of the proof, when $t$ is located on a regular point of $\partial \Omega$, is similar to Theorem 2. By contrast, the situation where $t$ is on a separating curve requires a different method than the one contained in the proof of Theorem 3 .

\section{Acknowledgements}

The authors acknowledge support from NSF grant DMS 1008900/1008907; D.L. also acknowledges support from NSF grant DMS (Career) 1005799.

\section{References}

1. E. Candès and D. Donoho, Continuous curvelet transform: I. Resolution of the wavefront set, Appl. Comput. Harmon. Anal. 19 (2005), 162-197.

2. T. Chan and J. Shen, Image Processing and Analysis, SIAM, Philadelphia, 2005.

3. M. Do Carmo, Differential geometry of Curves and Surfaces, Prentice Hall, 1976.

4. P. Grohs, Continuous shearlet frames and resolution of the wavefront set, Monatsh. Math., to appear (2011).

5. G. Easley, K. Guo, and D. Labate, Analysis of Singularities and Edge Detection using the Shearlet Transform, Proceedings of SAMPTA “09, Marseille 2009.

6. K. Guo, D. Labate and W. Lim, Edge Analysis and identification using the Continuous Shearlet Transform, Appl. Comput. Harmon. Anal. 27 (2009), 24-46.

7. K. Guo, and D. Labate, Characterization and analysis of edges using the continuous shearlet transform, SIAM Journal on Imaging Sciences 2(2009), 959-986.

8. K. Guo, and D. Labate, Analysis and Detection of Surface Discontinuities using the 3D Continuous Shearlet Transform, Appl. Comput. Harmon. Anal., 30 p. 231-242 (2011). 
9. K. Guo, and D. Labate, Characterization of Piecewise-Smooth Surfaces Using the 3D Continuous Shearlet Transform, submitted, 2011

10. S. Jaffard, Y. Meyer, Wavelet methods for pointwise regularity and local oscillations of functions, Memoirs of the AMS, 123 n.587 (1996).

11. S. Jaffard, Pointwise smoothness, two-microlocalization and wavelet coefficients, Publications Matematiques 35 (1991), p. 155-168

12. G. Kutyniok and D. Labate, Resolution of the wavefront set using continuous shearlets, Trans. Amer. Math. Soc. 361 (2009), 2719-2754.

13. C. Herz, Fourier transforms related to convex sets, Ann. of Math. 75 (1962), 81-92.

14. M. Holschneider, Wavelets. Analysis tool, Oxford University Press, Oxford, 1995.

15. S. Mallat, A Wavelet Tour of Signal Processing, Academic Press, San Diego, 1998.

16. Y. Meyer, Wavelets and Operators, Cambridge Stud. Adv. Math. vol. 37, Cambridge Univ. Press, Cambridge, UK, 1992.

17. G. Kutyniok and D. Labate, Resolution of the Wavefront Set using Continuous Shearlets, Trans AMS, 361 (2009), 2719-2754.

18. E. M. Stein, Harmonic Analysis: real-variable methods, orthogonality, and oscillatory integrals, Princeton University Press, Princeton, NJ, 1993.

19. S. Yi, D. Labate, G. R. Easley, and H. Krim, A Shearlet approach to Edge Analysis and Detection, IEEE Trans. Image Process 18(5) 929-941, (2009). 


\section{Index}

analysis of singularities, 1

using wavelets, 8

continuous shearlet transform (2D), 7, 10

continuous shearlet transform (3D), 31

localization lemma, 16

shearlet analysis of singularities corner points, 14

disc case, 10

Heaviside function, 6

linear delta, 2

step singularities (2D), 7

step singularities (3D), 32

singular support, 2

wavefront set, 2 